\title{
RFID READER ANTENNAS FOR TAG DETECTION IN SELF-CONFINED VOLUMES AT UHF
}

\author{
Carla R. Medeiros ${ }^{1}$, Jorge R. Costa ${ }^{1,2}$, Carlos A. Fernandes ${ }^{1}$ \\ ${ }^{1}$ Instituto Telecomunicações, Instituto Superior Técnico, Av. Rovisco Pais 1, 1049- \\ 001 Lisboa, Portugal, Tel: +351218418480, Fax: +351218418472 , \\ E-mails: \{carla.medeiros, jorge.costa, carlos.fernandes\}@1x.it.pt \\ ${ }^{2}$ ISCTE-IUL Instituto Universitário de Lisboa, Av. das Forças Armadas, 1649-026 \\ Lisboa, Portugal
}

Keywords: UHF Microstrip Antennas, RFID antennas, Near-field UHF RFID, Smart RFID reading surfaces.

\begin{abstract}
:
Confinement of the detection region is a critical issue for some important RFID applications where object coarse location is required along with its identification. It is a challenge at the UHF band to confine antenna radiation to reasonably sharp interrogation volumes $<10 \lambda^{3}$ without resorting to physical barriers. This paper presents a novel approach for RFID reader radiating structures that self-confine tag detection to a desired volume avoiding undesired readings outside the interrogation volume. The proposed solution covers simultaneously the three world assigned RFID frequency subbands at UHF. To demonstrate the effectiveness of the solution, the proposed configuration is integrated into bookshelves, a conveyor belt system, and a proximity point reader which form, together with appropriate control application, the building blocks of a smart store with automatic inventorying and billing capability. The radiating
\end{abstract}


structure concept is explored by full-wave simulations and it is validated both with nearfield measurements and with tag reading scores. Short demonstration videos are available on-line.

\section{Introduction}

Radio Frequency Identification (RFID) is a rapidly expanding technology that enables radio detection and recognition of objects associated to a univocal identification code carried by an electronic chip attached to an antenna - the RFID tag. These tags send back their univocal code whenever they are interrogated by an RFID reader within range. The basic concept is in use since the eighties, initially for automatic toll systems. However, the many advantages of this technology and the rapid dropping of associated manufacturing costs extended its applicability to other mass applications like pet or object identification, inventorying, asset management, contactless access control, anticounterfeiting and anti-theft systems, health care, etc [1]-[2]. Recently, RFID technology has been extended to allow simultaneously some degree of tag tracking and location [3]-[5].

RFID tags can be passive, retrieving the necessary energy from the interrogating wave, or can include a battery (active tags) to enhance reading range. Passive tags are the cheapest to manufacture, do not require maintenance and are more compact and lighter. World assigned RFID frequency bands range from High Frequency (HF) up to microwaves [1]-[2]. The allocated band at Ultra High Frequency (UHF) ranges from $860 \mathrm{MHz}$ to $960 \mathrm{MHz}$, divided into three sub-bands corresponding to the three world regions. In general, UHF passive RFID tags [6] present the lowest unit cost, in the order of cents, which makes UHF RFID the preferred choice for mass applications. Its cost is expected to decrease within a few years from 0.20 to 0.05 USD [7] making it affordable to attach to very low value products. It has potential to be used for instance on all items 
from retail stores and supermarkets (item-level tagging - ILT) to allow automatic realtime inventorying, tracking of misplaced items and unattended billing at cashiers, totally replacing the present manned optical bar code reading in every instance of store items and assets control. Unlike optical reading, RFID technology enables simultaneous multiple tag reading and does not require tag visibility.

The change of paradigm for item tracking in stores or warehouses requires the possibility to confine RFID tag reading to predefined volumes like those associated with individual shelves, cashier conveyor belts, proximity point readers, etc., without unwanted readings from immediate neighboring volumes. However, it is a challenge at UHF to limit detection to small sharp regions of the order of $<10 \lambda^{3}$ without resorting to intrusive and costly metal plates, absorbing shields or gates [8]. Unlike HF RFID, coupling between reader and tag antennas at UHF is made through a propagating electromagnetic wave which inherently enhances tag detection range.

This paper presents a very simple, effective and low-cost RFID solution for smart reading applications at UHF that allows retrieving tag information from objects placed on shelves, proximity point readers or sliding over conveyor belts. The proposed configuration overcomes the field confinement problem at UHF, intrinsically limiting tag detection to the boundaries of the region of interest, ensuring near-range univocal detection over the entire surface without the need for physical barriers. The structure is very simple, with mild fabrication tolerance, appropriate for extremely low-cost mass production. Furthermore, the solution can be easily attached to pre-existing store furniture, reducing cost and time required for technology change. To validate the proposed solution, prototypes of bookshelves, an isolated point reader and a cashier conveyor belt are characterized, fabricated and tested at the UHF band using commercially available RFID reader equipment and tags. The authors first proposed in 
[9] a preliminary RFID bookshelf solution and in [10] the smart conveyor belt configuration. A patent is pending [11] and a demonstration video can be seen on-line [12].

This paper is organized as follows. Section 2 states the volume confinement problem for UHF-RFID which is the motivation of the present paper. The subsequent section is dedicated to the characterization of the radiating structure proposed for smart shelf, proximity point reader and conveyor belt solutions for UHF RFID. Section 4 presents the fabricated prototypes and the corresponding experimental reading scores using commercial RFID tags and readers, demonstrating the effectiveness of the proposed solution. Conclusions are drawn in Section 5.

\section{Near-range RFID reading}

Coarse location can be accomplished by creating subareas with sharply confined reading range, without overlapping neighboring volumes. Tag location can then be identified through association with the interrogated volume. The main challenges in near-range UHF RFID are:

a) First, to ensure that the antenna reading capability is confined to the volume above the reading surface, that its coverage is uniform without blank spots to ensure $100 \%$ reading score and that it does not extend to undesired adjacent volumes; self-confinement must be sharp enough so that the reader can discriminate for instance adjacent shelves or adjacent cashier conveyor belts in supermarkets;

b) The solution must be compatible with the existing commercial passive UHF tags, which are much cheaper than active tags; it is desirable that the antenna operation band is wide enough to cover the three RFID world UHF bands; 
c) The reader antenna must be simple enough for very low-cost mass production and easy integration into different store structures shapes and sizes (shelves, tables, conveyors) inclusive into existing ones.

Fig. 1 shows the generic configuration of an RFID system that explores the proposed UHF RFID smart reading concept. A single reader can be used with multiple smart devices (shelves, tables, conveyors, point readers) using a standard electronically actuated RF switch to sequentially read each RFID smart device in the store. In this way, the RF power output from the single reader is enough to read an arbitrary number of smart devices in the store. A dedicated application is run on the computer to manage the reading sequence, data logging and data processing. Of course this configuration can be replicated several times in a large store.

Some solutions limited to RFID smart tables and shelves can be found in the literature, but most of these operate at the HF band [13]-[14]. The main difference to UHF is that energy coupling between the reader antenna and the tag at HF is essentially made through the magnetic field: coils or loops are typically used for the HF reader and tag [13]-[14]. The detection region is inherently very short, requiring in some configurations the repetition of the reading antenna element along the shelf length to enable its full coverage [15]. This becomes intrusive, adds complexity to the feeding circuit and increases cost. Moreover, HF passive tags are larger than UHF tags, have very low data rate and are more expensive.

Near-range UHF RFID antennas have been reported for tag detection in shallow regions with footprints of the order of $\lambda \times \lambda$ : for instance an inverted $L$ with a parasitic patch element is proposed in [16] but its use for smart shelves again requires multiple antennas to cover the whole length of the shelf, repeating the same problem identified above. An alternative solution based on multiple meandered open-circuited microstrip 
lines with slotted ground plane is proposed in [8]; the structure is moderately complex and no coverage results are presented for larger surfaces like those from typical shelves. As for conveyor belt applications, existing UHF RFID solutions employ patch type antennas [17], either as single elements combined with shielding barriers or as multiple elements arranged in a tunnel-like structure to force confinement of the radiated field [18]. Either configuration is very intrusive and inappropriate for store front-end.

The solution presented in this paper incorporates a single leaking microstrip line laid along the structure length, with an extended ground plane. Microstrip lines are commonly used at microwaves as transmission lines, due to its ease of fabrication and integration with other devices. However, depending on substrate parameters and strip shape, the leaking fields may be enhanced. Although undesired for usual microwave circuits [19], this leaking characteristic can be explored for the present RFID application to produce controlled close-range tag detection at UHF, without readings from items located outside the desired volume. One end of the microstrip line is connected to the reader and the other end to a matched load. It will be shown that this very simple solution fulfills all the requirements stated above.

\section{Radiating structure characterization}

This section describes in detail the basic radiating structure applied to a shelf and its modified meandered versions applied to a cashier conveyor belt and to an isolated point reader. These are three of the main equipments required for a RFID based retail shop. Full wave simulations and model optimizations are performed using the transient solver from CST Microwave Studio ${ }^{\mathrm{TM}}$ [20] based on Finite Integration Method. The concept is validated through RF measurements and using commercial RFID readers and tags to assess the reading score. 


\subsection{RFID Smart Shelf}

An example of smart shelf structure [9] is shown in Fig. 2. It uses a straight microstrip line running across the shelf length along the $\mathrm{x}$-direction. The microstrip line is probe fed at one end and it is terminated on the other with a matched load.

It is well known that the main electric field lines in a microstrip flow from the metal strip to the ground plane through the substrate layer, while some radiation leaks to the air as show in Fig. 3. Low permittivity styrofoam $\left(\varepsilon_{\mathrm{r}} \approx 1.05\right)$ substrate layer is used to enhance these leaking fields. The electric field orientation is mainly transversal to the microstrip line. Although this places a restriction on tag orientation for reliable detection it is just perfect for bookshelf application, where books tend to be placed according to a preferred orientation. As will be seen ahead, this is not a limitation of the concept since some meandering of the strip path is enough to enable arbitrary tag orientations reading.

Prototype overall dimensions are typical for bookshelves, $30 \mathrm{~cm} \times 100 \mathrm{~cm}$. Substrate thickness is $1 \mathrm{~cm}$ and transmission line width is $5 \mathrm{~cm}$, leading to $50 \Omega$ line characteristic impedance. The line is fed by a $50 \Omega$ feeding coaxial cable (EZ-141-AL) and it is terminated by a $50 \Omega$ resistor. The strip width, along with the fact that the ground plane is extended up to the edges of the shelf contribute to obtain the adequate field distribution above the shelf without significant spillover to the volume below. Vertical decay of the fields also ensures isolation with respect to an upper shelf. Depending on the shelf size, other combinations of strip width and substrate thickness may be more favorable, eventually leading to different value of the characteristic impedance. The relation between strip width and substrate thickness is determined by the known equation of the microstrip characteristic impedance [21]. The higher the substrate thickness the higher is vertical detection. The width of the detection range 
within the bookshelf is mainly controlled by the width of the ground plane. In fact, it is possible to ensure tag detection up to a bookshelf width of about 7 to 8 times the width of the microstrip line, as long as the ground plane covers the entire bookshelf bottom face. A word of caution is worth if the strip is chosen too wide, because the null of the transverse E-field component along its axis may compromise tag detection over this line.

Although not required, in the presented example an additional layer of styrofoam substrate is added on top of the transmission line to increase mechanical stability and protect the radiating structure from the contact with the books.

To evaluate the shelf performance and analyze the electric field behavior, near-field calculations were performed for the UHF band (at $866 \mathrm{MHz}$ ). Considering the coordinate frame from Fig. 2, shelf boundaries correspond to $z= \pm 15 \mathrm{~cm}, x= \pm 50 \mathrm{~cm}$ and the shelf surface corresponds to $y=0$. The electric field amplitude distribution in the $y z$ and $x y$ planes for $1 \mathrm{~W}$ RF source power is shown in Fig. 4. The results at the $x=0$ plane, shown in Fig. 4a) demonstrate that the leaking fields are strong above the shelf, reasonably confined to the near-zone, and dominant electric field components are $E_{y}$ and $E_{z}$. The intensity of the latter component is low at the centre of the shelf, conversely to the vertical component. At the shelf surface, Fig. 4b) $y=0$ plane, the intensity of the $E_{y}$ and $E_{z}$ components is reasonably constant within the shelf area and decrease rapidly outside the shelf boundaries. Experiments have shown that the minimum field level for detection of a common commercial tag, which is described in the next section, is of the order of $20 \mathrm{dBV} / \mathrm{m}$. So, Fig. 4 anticipates that tag detection will be well confined to the top surface of the shelf, preventing detection outside the interrogated shelf without the need of EM isolation barriers. Experimental results shown ahead will confirm this. 
It is interesting to analyze how the presence of books affects the previous field distribution. Simulations were repeated with 21 books placed on the shelf. The books were modeled as $0.5 \mathrm{~cm}$ spaced blocks of $\varepsilon_{r}=2.3$ dielectric, with $4 \mathrm{~cm}$ width, $23 \mathrm{~cm}$ height and $18 \mathrm{~cm}$ depth. The result is presented in Fig. 5 and it shows that the field intensity is enhanced everywhere in the shelf, inclusive into the books as if they drain power out from the transmission line. Even the $E_{\mathrm{x}}$ component is greatly enhanced. Nonetheless, results demonstrate that the total electric field $E_{t}$ remains at low level outside de shelf compared to field level over the shelf.

Simulated results from Fig. 4 and Fig. 5 suggest that circular polarized or dual polarized tags are the preferred choice for detection of arbitrarily oriented objects above the shelf. However, full detection is also ensured with linear polarized tags placed parallel to the $y z$ plane; other orientations may reduce tag reading score in this case. This will be analyzed ahead.

Fig. 6a shows two manufactured shelf prototypes and the monitoring application screen. Each shelf comprises a wooden rectangular frame for mechanical support and two sheaths of plywood which are assembled to form a hollow structure that houses the microstrip line and the styrofoam substrate and superstrate. Details of the microstrip feeding point and of the termination can be seen in Fig. 6b and Fig. 6c. The reader is located outside the shelf, according to the scheme of Fig. 1, but a smaller commercially available reader module [21] can be accommodated inside the shelf along with the proposed antenna to obtain a stand-alone smart shelf solution.

Measured and simulated input return loss of the embedded straight transmission line is presented in Fig. 7, both for the empty shelf and when it is fully loaded with 21 books. The agreement between simulation and measurements is quite satisfactory. It is noted that when the 21 books are present, the line input return loss improves slightly, 
consistent with previous interpretation that more power is drained out from the line in this case. It is noted that the operation band covers simultaneously the three world assigned RFID sub-bands at UHF, bounded by the two vertical dashed lines.

Near-field measurements of the empty shelf were performed using Agilent PNA E8361A network analyzer with a small electric field probe. Near-field was scanned across two cut planes: $z$-axis scan (plane $x=0, y=0$ ) and vertical scan (plane $x=0$ and $z=3$ ). Measured results of $E_{y}, E_{z}$ and total electric field, shown in Fig. 8 and Fig. 9, demonstrate good agreement with simulations. Results for the $E_{x}$ component are not shown because its amplitude is very low. Consistent with simulation results in Fig. 4, the transversal scan from Fig. 8 shows faster field intensity decay outside the shelf boundaries (beyond the ground plane, marked with vertical dashed lines). This shows the relevance of extending the ground plane towards the shelf boundary. Fig. 9 shows the total electric field magnitude measured across the closed ABCD contour, drawn 10 $\mathrm{cm}$ apart from the shelf boundary (at $\mathrm{Y}=0$ ); the results demonstrate that the total electric field remains at low level compared to the field level over the shelf and in fact mostly below the referred $20 \mathrm{dBV} / \mathrm{m}$ threshold. Fig. 10 shows the vertical decay of the fields, confirming that the predicted extension of the vertical coverage.

No measurements of this type are shown for the shelf loaded with books, but the demonstrated good agreement between previous simulations and measurements allows trusting the results presented in Fig. 5. Nevertheless, experimental tag detection scores are thoroughly analyzed ahead both for this case and for the empty shelf.

\subsection{RFID Smart Conveyor Belt}

The overall width and length of the studied conveyor belt structure is $40 \mathrm{~cm} \times 120$ $\mathrm{cm}$, typical dimensions for cashier application. The same type of radiating structure is 
proposed for integration with the conveyor belt as shown in Fig. 11. In this case the transmission line is slightly meandered to enhance the leakage of the previous less dominant field components and to extend tag detection height for all tag orientations in a controlled way [10]. As before, $1 \mathrm{~cm}$ thick styrofoam substrate is used. The width of the microstrip line is $5 \mathrm{~cm}$, leading to $50 \Omega$ characteristic impedance. The meandered line is probe fed at one end and terminated at the other end with a matched load (50 $\Omega$ resistor). The microstrip line and ground plane $(30 \mathrm{~cm} \times 100 \mathrm{~cm})$ are cut from thin metal sheet. This is smaller than the size of the conveyor belt to fit inside it, mounted directly below the belt as shown in Fig. $11 \mathrm{~b}$.

To evaluate the performance of the proposed solution, near-field calculations were performed at the UHF band. Referring to the coordinate frame from Fig. 11, the embedded structure boundaries are $\mathrm{z}= \pm 15 \mathrm{~cm}, \mathrm{x}= \pm 50 \mathrm{~cm}$. Belt surface corresponds to $y=0 \mathrm{~cm}$. CST [20] was used again to calculate the volume distribution of the electric field components above an empty conveyor belt for $1 \mathrm{~W}$ power fed to the microstrip line. The results are presented in Fig. 12. It is clear that the presence of the meanders significantly enhances all the near-field components (Fig. 12a) while still remaining confined to the structure boundary. However the field distribution at the conveyor surface presents isolated low field spots near the edges of each meander of the microstrip line. This is not a setback because conveyor belt continuous movement and consequent travel of the objects over the microstrip line is just appropriate to ensure that the tag is exposed to different field values and orientations originated in different parts of the meandered line. Thus, tags travelling over the device at least for one period of the meander can be detected for any arbitrary orientation, provided they are exposed to the appropriate field level during the travel. 
Given the above, Fig. 12b) shows for each $(y, z)$ tag position the maximum $E$-field value that can be found when scanning the $x$ coordinate in the $[-50 \mathrm{~cm}, 50 \mathrm{~cm}]$ interval. The results show that $E_{z}$ is still the dominant leaking electric field component but it now extends well above the belt surface. Yet, $E_{x}$ and $E_{y}$ field components are also enhanced, although decreasing with height faster than $E_{z}$. All field components are reasonably confined to the shelf near-zone, preventing detection of adjacent conveyor belts without the need for isolation barriers.

The fabricated prototype is shown in Fig. 13. A thin acrylic layer was glued on the top face of the microstrip assembly to favour smooth sliding of the belt over it. Neither the acrylic nor the belt layers affect the antenna performance.

The input return loss of the conveyor belt prototype was measured and results agree well with the meandered line simulations, Fig. 14. It is seen that the device can operate simultaneously with any of the three world assigned RFID sub-bands at UHF bounded by the two vertical dashed lines. Tag reading scores for this structure are presented ahead.

\subsection{RFID Smart Point Reader}

Large retail shops and stores usually provide individual optical bar-code readers to enable users to self-check item prices. It is possible to extend this concept for RFID tagged item. For this application, the RFID reader must be able to read tags with any orientation, while confining the detection to avoid unwanted reading of neighboring tags. Desirably, it should allow reading simultaneously more than one item held by the user in a single scan. A point reader can be easily obtained by appropriately reducing the total length of the meandered microstrip line and the width of the ground plane, as shown in Fig. 15. A demonstration example was designed and optimized using a $1 \mathrm{~cm}$ 
thick styrofoam substrate and $5 \mathrm{~cm}$ width microstrip line to obtain $50 \Omega$ characteristic impedance. The overall dimensions are $27.5 \mathrm{~cm} \times 22 \mathrm{~cm}$.

The near-field distribution at the isolated point reader surface is shown in Fig. 16. The amplitude of all field components is very similar to what was found for the conveyor belt, reasonably confined to the reader near-zone. The $y z$ plane simulations (not shown) anticipate tag detection up to $15 \mathrm{~cm}$ above the structure. Fig. 17 shows the fabricated prototype, wherein the meandered microstrip line is encased in a shallow acrylic box.

\section{RFID UHF tag reading score}

To evaluate the effectiveness of the proposed structures, experimental tag reading score tests were performed with the setup shown in Fig. 18a). The ALIEN - ALR-8800 reader [22] (Fig. 18b) was used to feed $1 \mathrm{~W}$ to all the proposed smart devices through a routing switch as indicated in Fig. 1. ALIEN native software was used to determine tag reading score. Commercial UHF tags were selected for the test: ALIEN - ALN-9540 "Squiggle" GEN 2 tag [22], Fig. 18c). Its size is $9.7 \mathrm{~cm} \times 1.1 \mathrm{~cm}$, the polarization is linear and it operates from $860 \mathrm{MHz}$ up to $920 \mathrm{MHz}$.

Next we present reading scores for each of the developed devices. Videos with experimental demonstrations of these devices can be found in [12].

\subsection{RFID Smart Shelf}

Three different score tests were performed for the smart shelf structure [9]. In one test the reading score was evaluated for a stand-alone tag that was positioned successively at different uniformly distributed locations in the volume above the shelf. The second test was performed in a similar way, but with the tag attached to a book. In 
the third test, multiple books with attached tags were ranged across the shelf to evaluate the simultaneous reading score.

Fig. 19 shows experimental reading scores for the first type of tests with the tags oriented along the z-axis above the shelf. A large number of tag interrogations were performed automatically for each tag position and the corresponding percentage of detections was recorded. Filled circles indicate the positions where the tag exhibited $100 \%$ detection, while the cross marker " $\mathrm{x}$ " indicates the positions without detection. Each dot corresponds to the center of the tag. Fig. 19 shows full tag detection over the shelf with good lateral isolation. The height above the shelf surface where the tag can be detected is controlled by adjusting the RF signal attenuation on the reader. With full 1 $\mathrm{W}$ feed power, $100 \%$ detection score is obtained up to $7 \mathrm{~cm}$ above the shelf surface, without undesired detection of tags placed below the interrogated shelf or above the adjacent upper shelf. The $7 \mathrm{~cm}$ maximum detection height is sufficient for the bookshelf application. Although the $E_{z}$ component intensity is very low at the centre of the microstrip line, this effect is much localized at $z=0$ and does not deter reliable tag detection. It is mentioned for reference that tags were detected where simulations predicted field levels above $20 \mathrm{~dB} \mathrm{~V} / \mathrm{m}$.

In the second type of reading score tests, the tag was attached to the inner side of the back cover of a book, near its bottom part. The book was placed up-right at different positions above the shelf with the tag parallel to the $\mathrm{z}$-axis. This is the preferred orientation to take advantage of the predominant and well confined $E_{z}$ field component. Results are shown in Fig. 20a). Each dot corresponds to the position of the center of the book. As before, filled circles indicate the positions where the tag exhibited $100 \%$ detection, while the cross marker " $\mathrm{x}$ " indicates the positions without detection. 
Book detection is reasonably contained within the limits of the shelf for z-axis tag orientation. Yet, experiments showed that near $100 \%$ detection score was still possible when the tag inside the book was rotated in the $y z$ plane. Even for the extreme y-axis tag orientation inside the book, detection was always possible over most of the shelf surface, except in a narrow strip located about $7 \mathrm{~cm}$ apart from the centre of the shelf (marked with unfilled circles in Fig. 20b) where $E_{y}$ amplitude is low and tag reading is unreliable. Unfilled circles thus indicate positions where the tag reading was intermittent. Experimental results (not shown) confirm detection also for $x$-axis tag orientation ( $E_{x}$ component in Fig. $5 \mathrm{~b}$ ) above the microstrip line, where the other electric field components are also significant.

So, books must be preferably placed in the up-right position on the shelves, with the respective tags attached to the bottom of the cover along the z-axis. Nonetheless, good flexibility is allowed for tag orientation and for its height in the book, without compromising reading score. This flexibility is attractive for practical use of the proposed solution. Similar tests were performed for multiple books, either arranged in clusters or fully loading the shelf. The previously presented conclusions for the single book at different positions on the shelf hold equally for these cases.

\subsection{RFID Smart Conveyor Belt}

A slightly different approach was adopted for the validation of the smart conveyor belt performance [10]. As previously referred, fully reliable detection within a reasonable height above the belt requires that the tagged items slide over the device. So, several tags were uniformly distributed in one transversal plane above the belt. Then, the cumulative reading score at UHF was registered for each tag $\left(y_{\mathrm{n}}, z_{\mathrm{n}}\right)$ as it slide along 
$x$ using the conveyor movement. Several complete $x$-sweeps along the belt length were performed in order to verify repeatability of the results.

Results obtained in this way are represented in Fig. 21. Filled circles correspond to $100 \%$ tag detection; unfilled circles correspond to tags detected only for some of the sweeps, intermittent readings. The cross marker " $\mathrm{x}$ " indicates failed tag detection for all the $x$-sweeps. Color and size of the filled circles gives an additional measure of the detection redundancy (larger circles correspond to higher number of times that the same tag was detected by the reader in a single $x$-sweep). The results demonstrate the effectiveness of tag detection and the good detection confinement to a small volume above the conveyor belt structure, as predicted by simulations. Again, tags were detected where simulations predicted field levels above $20 \mathrm{~dB} \mathrm{~V} / \mathrm{m}$. The maximum detection height with $100 \%$ score for all tag orientations and positions above the belt surface is $15 \mathrm{~cm}$, although detection height can reach $30 \mathrm{~cm}$ at certain spots for $x$ - and $y$ oriented tags or $100 \mathrm{~cm}$ for z-oriented tags. Fine control of the maximum detection height can be achieved through the reader output power control.

Tests were repeated with multiple tagged objects, placed isolated, in clusters, stacked, etc. Similar results were obtained, with $100 \%$ reading score ensured for items placed up to $15 \mathrm{~cm}$ above the conveyor belt for all orientations, not deterring detections at higher item positions for certain tag orientations. Lateral confinement of the detection was perfectly compatible with normal distances between parallel cashiers in retail shops and supermarkets. This already effective solution can be further redesigned to improve the detection range and to enhance the less intense field components if required for other applications. 


\subsection{RFID Smart Point Reader}

Tag reading tests were also performed with the isolated point reader. Since the tagged items are manually scanned in front of the device by the user, tag reading score study versus tag orientation is not relevant. The user intuitively adjusts the items orientation for best detection. The maximum detection range achieved experimentally for up to three simultaneously scanned tagged books was about $15-20 \mathrm{~cm}$. This value ensures good isolation with respect to neighboring smart furniture.

\section{Conclusions}

This paper presents new low-cost UHF RFID radiating structures that can be used with existing commercial readers to enable reliable tag detection in selective selfconfined interrogation volumes. The proposed approach uses in a controlled way the leaking fields from shaped microstrip transmission lines. It was demonstrated that good isolation is achieved with respect to non-desired adjacent regions without the need of physical barriers.

Prototypes of shelves, a cashier conveyor belt and a point reader were fabricated and tested together, emulating a retail shop environment. Test results using commercial reader and tags demonstrate a very good match between experimental reading score and the simulated near-field distribution. For the smart shelf prototypes, 100\% reading score was achieved with the books and tags in the usual preferred orientation. But other orientations are also allowed, which makes the proposed solution adequate for practical application on retail stores. For the conveyor belt system also $100 \%$ reading score was achieved for tagged items placed with arbitrary orientation up to $15 \mathrm{~cm}$ above the belt surface. Lateral confinement of the fields is very effective for all tag orientations which is appropriate for close parallel cashier conveyor operation in scenarios like 
supermarkets without cross-readings. Experimental tests of the point reader (not shown here, but available in [12]) also confirm the predicted performance, allowing to read simultaneously more than one scanned object.

The presented straight and meandered microstrip line structures can be redesigned, without frequency restriction, to adjust for different device sizes, materials and coverage requirements. The transmission line circuit can be embedded on the structures as demonstrated in this paper or can be just assembled in a self-contained layered structure to be laid on existing store furniture to reduce the cost of the technology change. The very interesting point to stress is the simplicity of the radiating structure and its mild fabrication tolerance, requiring no sophisticated fabrication environment. The microstrip line can be implemented for instance as metalized adhesive tape. Thus the concept is adequate for extremely low cost mass production at non-specialized facilities. In fact, a pilot experience has started in the sequence of the present work, where the radiating structure is being fabricated and integrated directly in a store furniture factory. A patent was filed [11].

Even though the presented work is focused on shelves, conveyor belts and point readers, the concept can been readily extended for other applications where tight detection volume confinement at UHF is required. It can replace some functions that are presently performed with optical bar code manual readers, enabling more efficient complete RFID based solution in retail shop environment.

\section{Acknowledgments}

The authors would like to thank Creativesystems for discussions about the motivation and system specifications and for providing the commercial RFID tags and reader equipment. Authors also thank V. Fred and C. Brito for prototype fabrication, A. Almeida for prototype measurements and E. B. Lima for designing the "RFID Store 
Manager" software for system control, data logging and information processing. The work was partly funded by Fundação para a Ciência e Tecnologia, through project RFID-Local PTDC/EEA-TEL/102390/2008.

\section{References}

[1] V. Chawla and Dong Sam Ha, "An Overview of Passive RFID," IEEE Communication Magazine, 4, September 2007, pp. 11-17.

[2] K. Finkenzeller, RFID Handbook, New York, Wiley \& Son, Inc., 2000.

[3] J. Hightower, R. Want, and G. Borriello, "SpotON: An indoor 3D location sensing technology based on RF signal strength," Univ. of Washington, Dep. of Comp. Science and Eng., WA, Seattle, Technical report, February, 2000.

[4] L. M. Ni, Y. Liu, Y. C. Lau, and A. P. Patil, "LANDMARC: indoor location sensing using active RFID," 1st IEEE International Conference on Pervasive Computing and Communications (PerCom '03) Digest, Texas, USA, March 2003, pp. $407-415$.

[5] P. Bahl, and V.N. Padmanabhan, "RADAR: An inbuilding RF-based user location and tracking system," 19th Annual Joint Conference of the IEEE Computer and Communications Societies (IEEE INFOCOM 2000) Digest, 2, Tel Aviv, Israel, March, 2000, pp. 775-784.

[6] K. Rao, P. Nikitin, S. Lam, “Antenna Design for UHF RFID Tags: A Review and a Practical Application," IEEE Transactions on Antennas and Propagation, AP53, 12, December, 2005, pp. 3870-3876.

[7] M. Lieshout, L.Grossi, G. Spinelli, S. Helmus, L. Kool, L. Pennings, R. Stap, T. Veugen, B. der Waaij, and C. Borean, "RFID Technologies: Emerging Issues, 
Challenges and Policy Options," Institute for Prospective Technological Studies, European Commission, Tech. Report, EUR 22770 EN, 2007.

[8] W. Choi, "Near-field antenna for RFID smart shelf in UHF," in Proc. IEEE International Symposium on Antennas and Propagation, June, 2009.

[9] C. R. Medeiros, J. R. Costa, and C. A. Fernandes, "RFID Smart Shelf with Confined Detection Volume at UHF," IEEE Antennas and Wireless Propagation Letters, 7, October, 2008, pp. 773-776.

[10] C. R. Medeiros, J. R. Costa, and C. A. Fernandes, "UHF RFID Smart Conveyor Belt with Confined Detection Range," IEEE International Symposium on Antennas and Propagation Digest, June, 2009.

[11] C. R. Medeiros, J. R. Costa, and C. A. Fernandes; PT 104121, June 2008 and PCT 2009000035 [Pending].

[12] UHF-RFID Self-confined Volume Detection, available at http://www.it.pt/auto_temp_web_page_preview.asp?id=666, December, 2009.

[13] X. Qing and Z. N. Chen, "Proximity Effects of Metallic Environments on High Frequency RFID Reader Antenna: Study and Applications,” IEEE Transactions on. Antennas and Propagation, AP-55, 11, November, 2007, pp. 3105-3111.

[14] A. Cai, X. M. Qing, and Z. N. Chen, "High Frequency RFID Smart Table Antenna," Microwave Optical and Technology Letters, 49, 9, September, 2007, pp. 2074-2076.

[15] X. Qing; Z. N. Chen, "Characteristics of a Metal-Backed Loop Antenna and its Application to a High-Frequency RFID Smart Shelf," IEEE Antennas and Propagation Magazine, 51, 2, April, 2009, pp. 26-38. 
[16] C. Cho, J. Ryoo, I. Park, and H. Choo, "Design of an RFID Reader Antenna for Near-Field Communications Using Opposite-directed Currents," Asia Pacific Microwave Conference Digest, Hong Kong and Macau, China, December, 2008.

[17] J. M. Lee, N. S. Kim and C. S. Pyo, "A Circular Polarized Metallic Patch Antenna for RFID Reader," Asia-Pacific Conference on Communications Digest, Australia, October, 2005, pp 116-118.

[18] Z.-M. Liu and R. R. Hillegass, "A 3 Patch Near Field Antenna for Conveyor Bottom Read in RFID Sortation Application," IEEE International Symposium on Antenna and Propagation Digest, July, 2006, pp. 1043-1046.

[19] D. M. Pozar, Microwave Engineering, 2nd Edition, New York, John Wiley \& Sons, Inc., 1998.

[20] CST- Computer Simulation Tech. website, available at http://www.cst.com/, December 2009.

[21] S. Ramo, J. Whinnery, and T. Duzer, Fields and Waves in Communication Electronics, 3rd Edition, New York, John Wiley \& Sons, Inc., 1994, p. 412.

[22] Skyetek - Embedded RFID Readers, available at http://www.skyetek.com/, December, 2009.

[23] ALIEN Technology website, available at http://www.alientechnology.com/, December, 2009. 


\section{List of Figures}

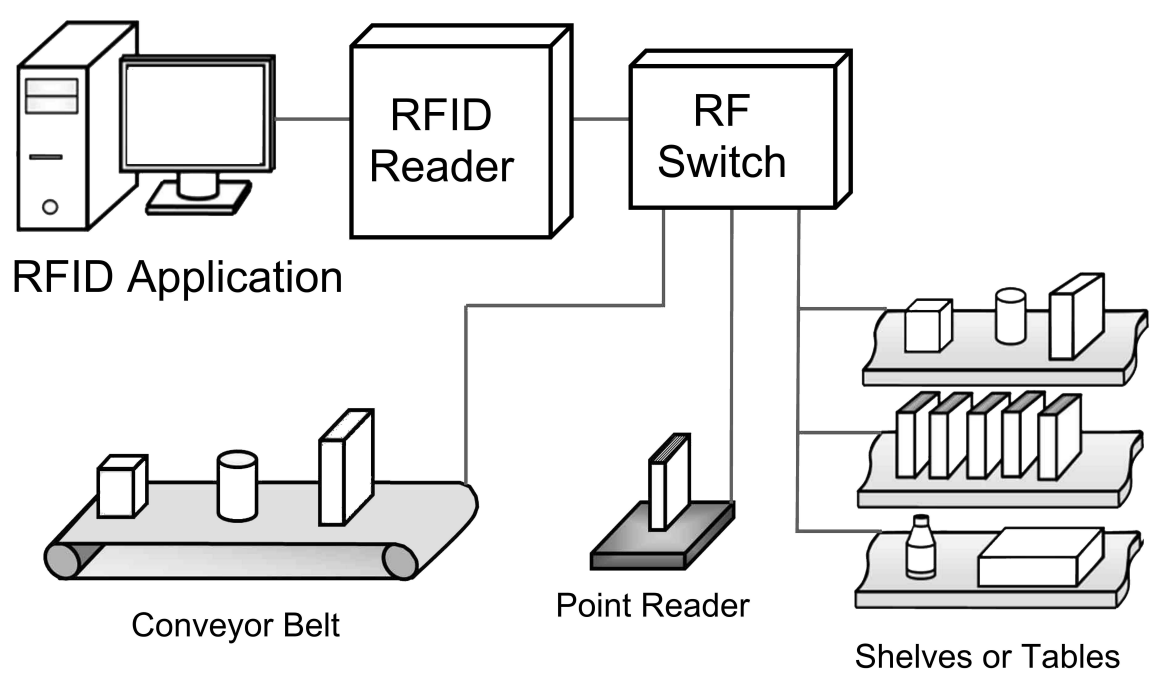

Fig. 1 RFID based smart reading system configuration for retail shop.

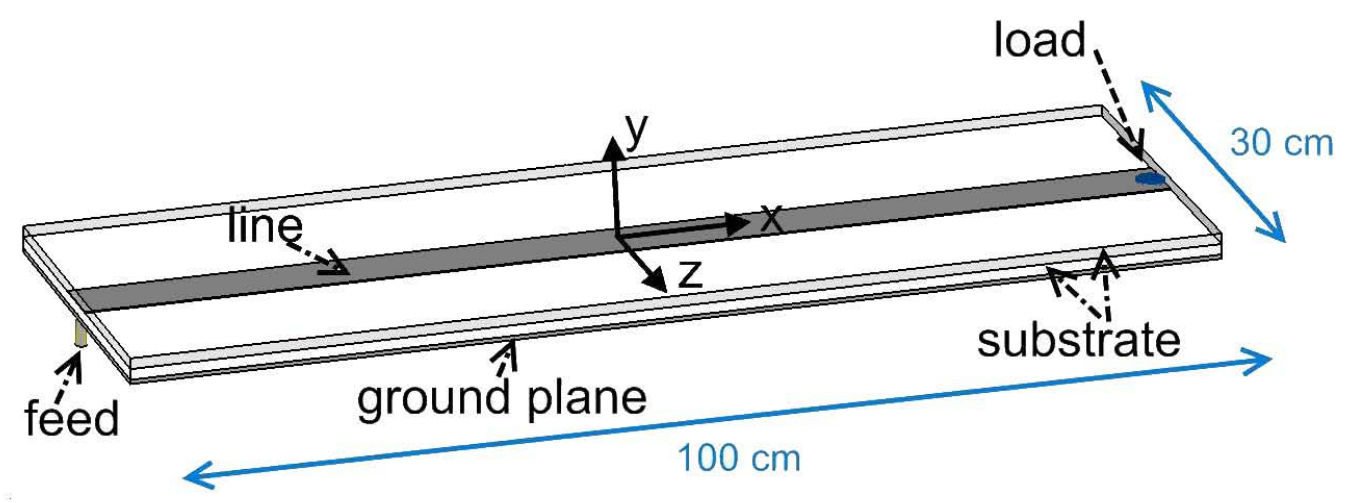

Fig. 2 RFID smart shelf configuration.

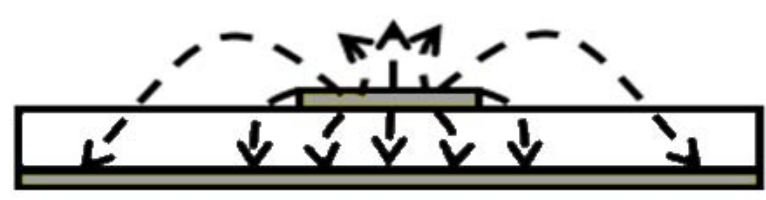

Fig. 3 Microstrip line electrical field behavior. 

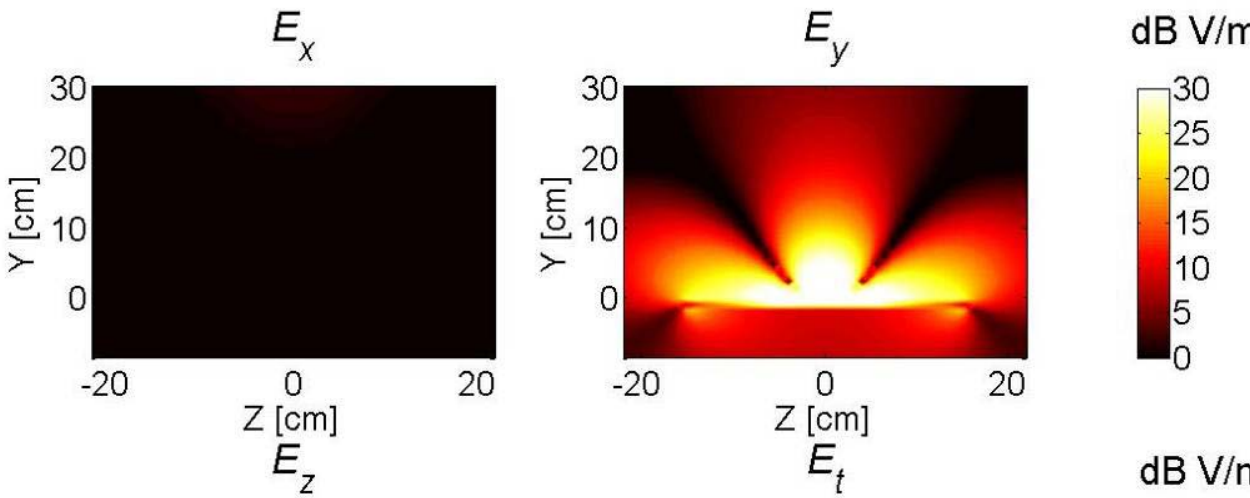

a)
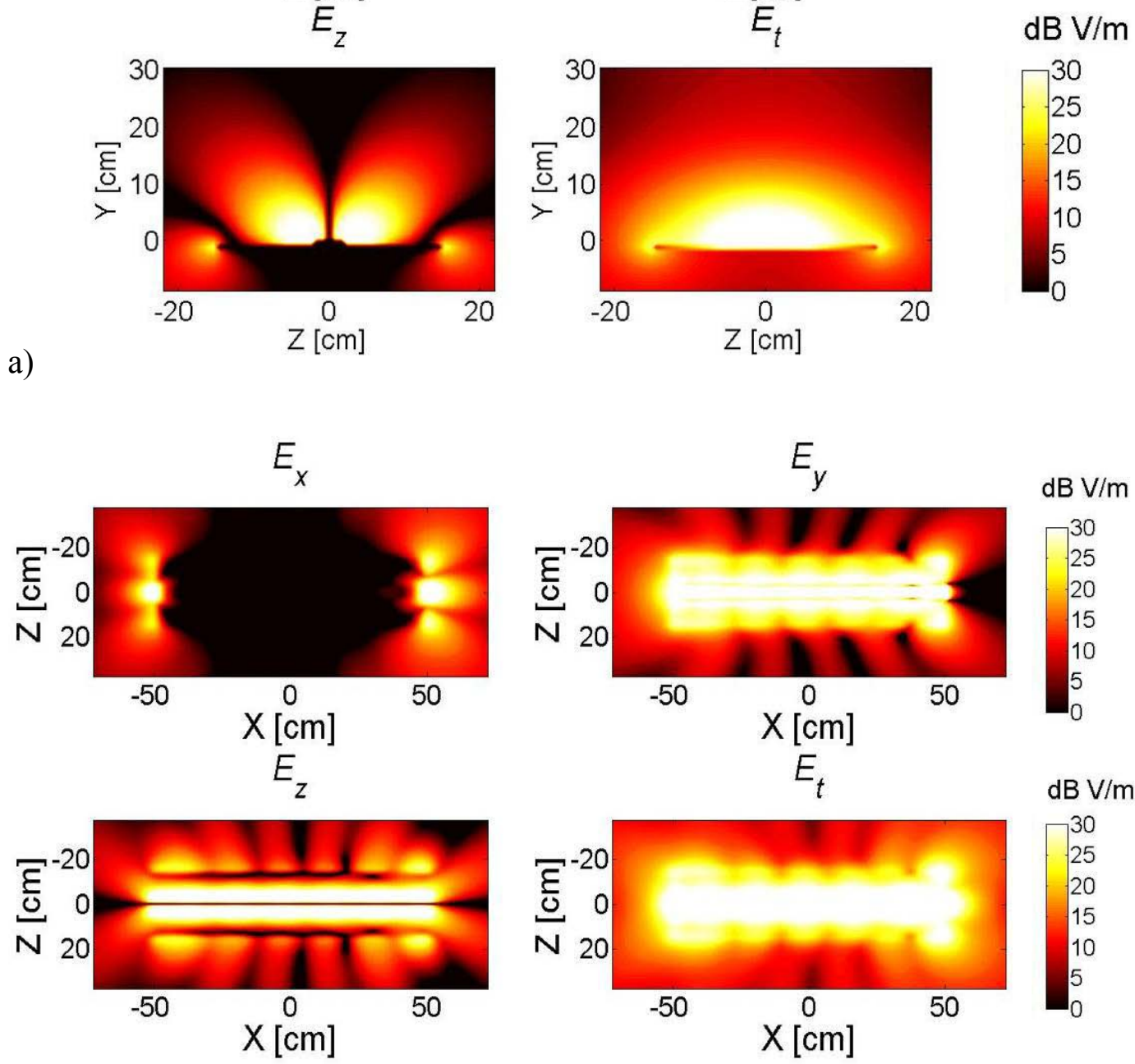

b)

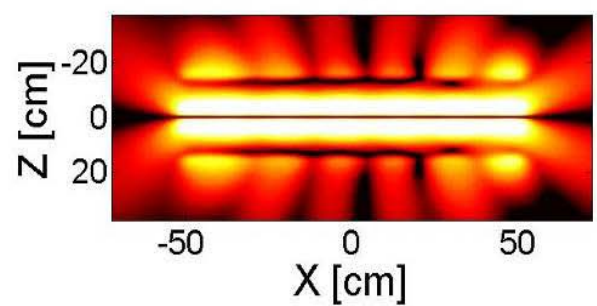

Fig. 4 Simulated near-field components $\left(E_{x}, E_{y}\right.$ and $\left.E_{z}\right)$ and total near-field $\left(E_{t}\right)$ amplitude distribution at $866 \mathrm{MHz}$ over an empty shelf: (a) $y z$ plane; (b) $x z$ plane, shelf surface. 

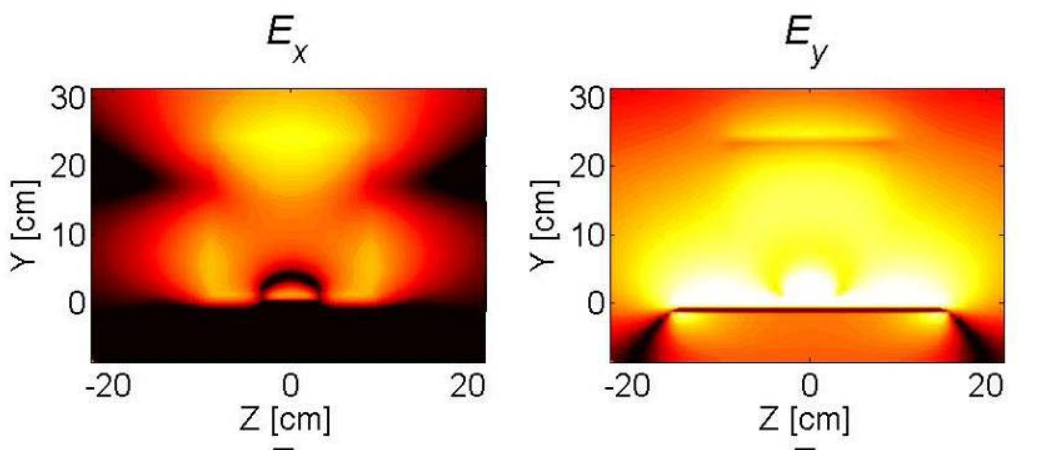

$\mathrm{dB} \mathrm{V} / \mathrm{m}$
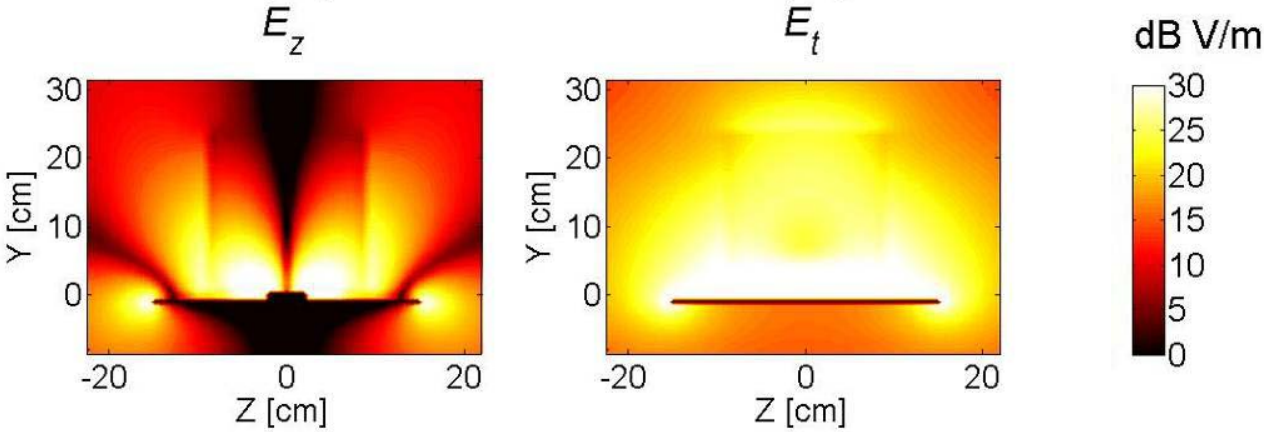

a)
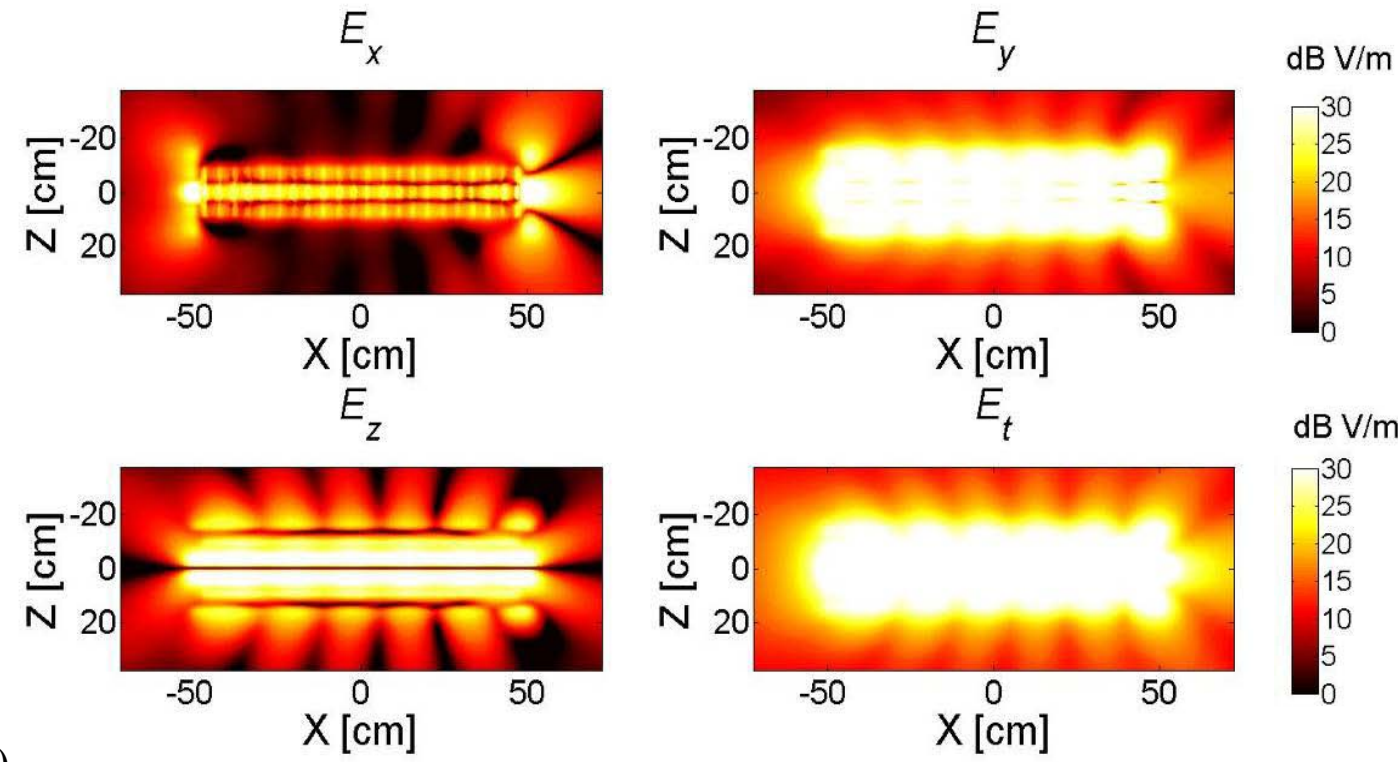

Fig. 5 Simulated near-field components $\left(E_{x}, E_{y}\right.$ and $\left.E_{z}\right)$ and total near-field $\left(E_{t}\right)$ amplitude distribution over the shelf at $866 \mathrm{MHz}$ when loaded with 21 books: (a) $y z$ plane; (b) $x z$ plane, shelf surface. 
a)
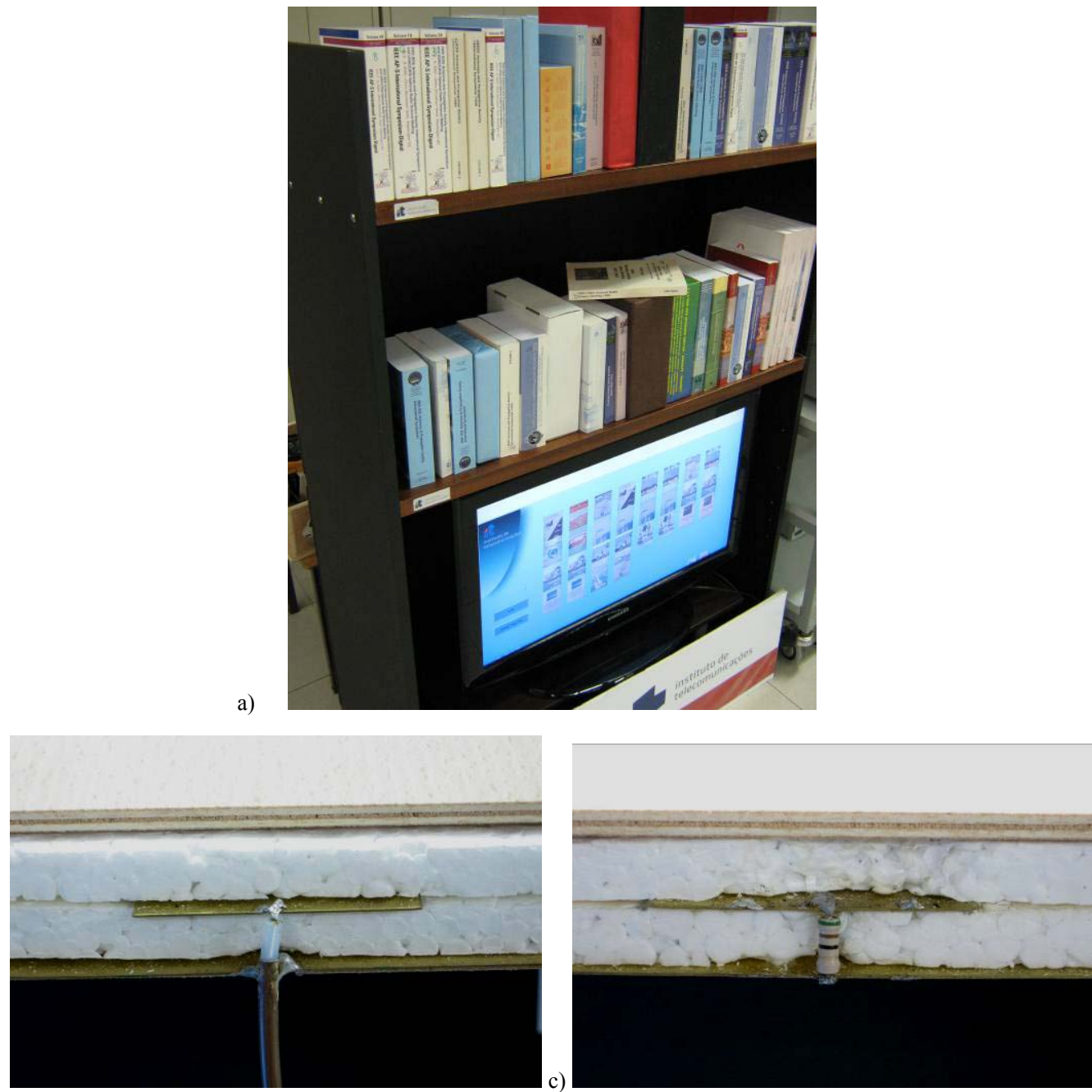

Fig. 6 Smart shelf: (a) Finished fabricated prototype and application screen; (b) Opened edge of the shelf showing detail of the line feeding point (c) Detail of the line termination.

a)
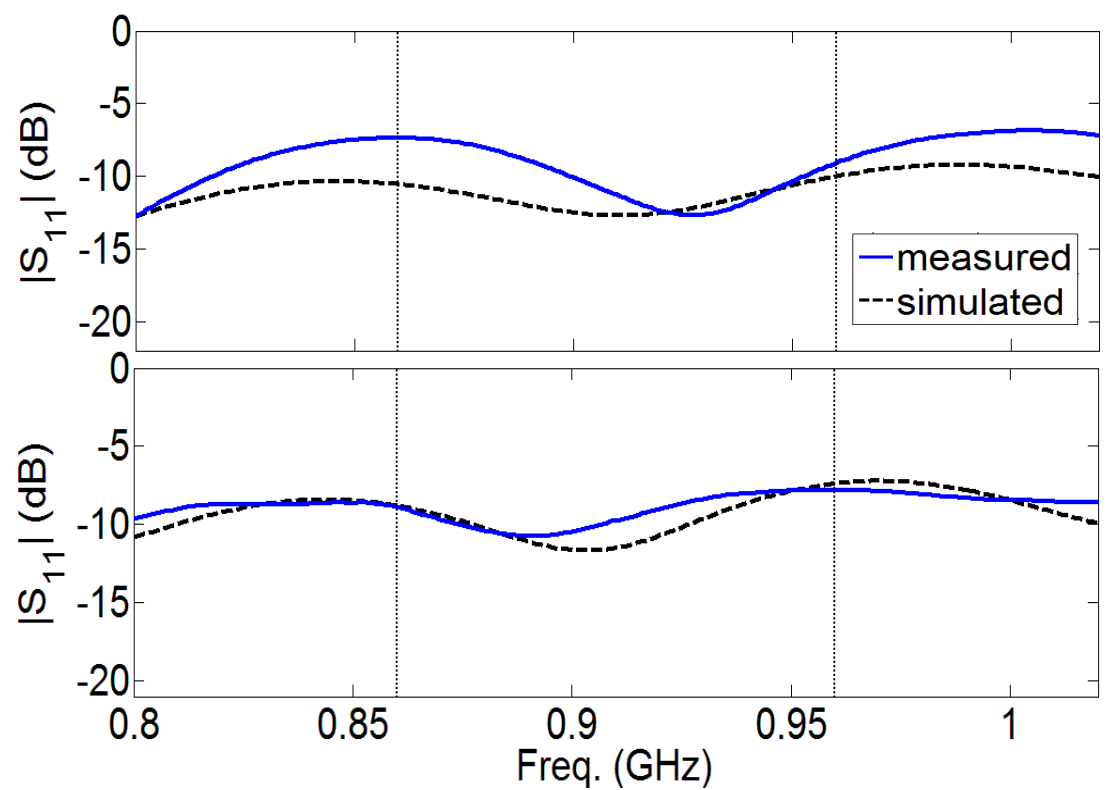

Fig. 7 Measured and simulated reflection coefficient amplitude: (a) empty shelf; (b) shelf fully loaded with 21 books. 

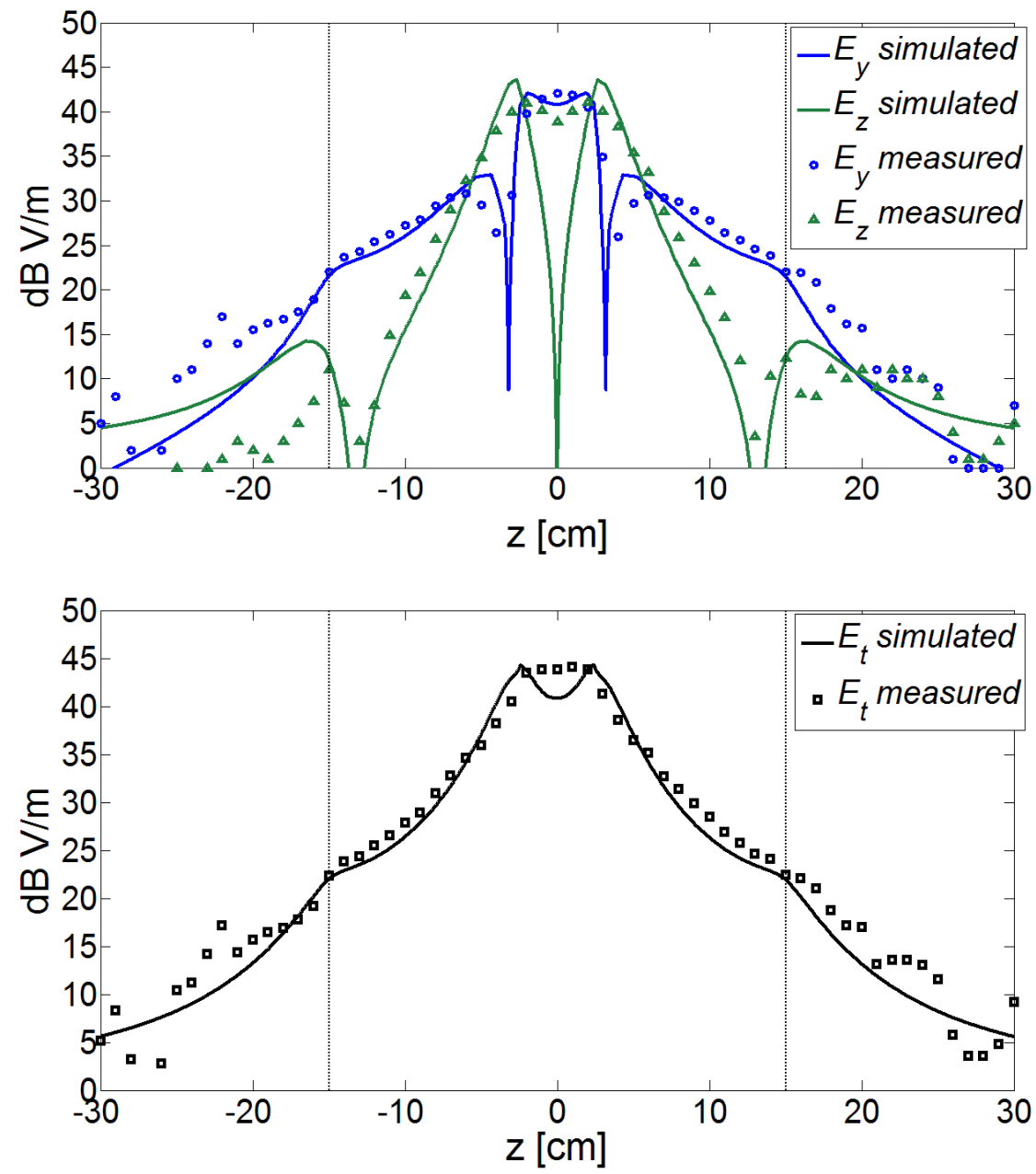

Fig. 8 Measured and simulated near-field components and total near-field amplitudes at the $(x=0, y=0)$ plane of the empty shelf.

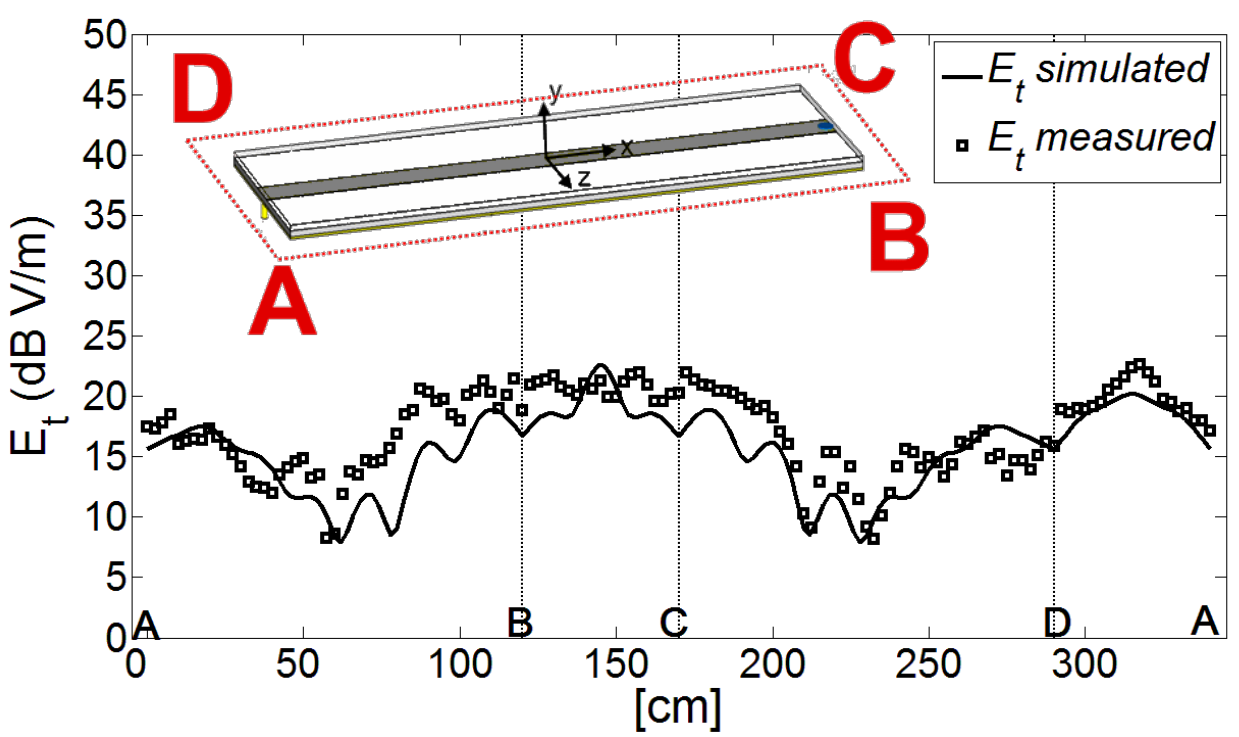

Fig. 9 Measured and simulated total electric near-field around the shelf at a distance $10 \mathrm{~cm}$ away from the shelf boundary. 

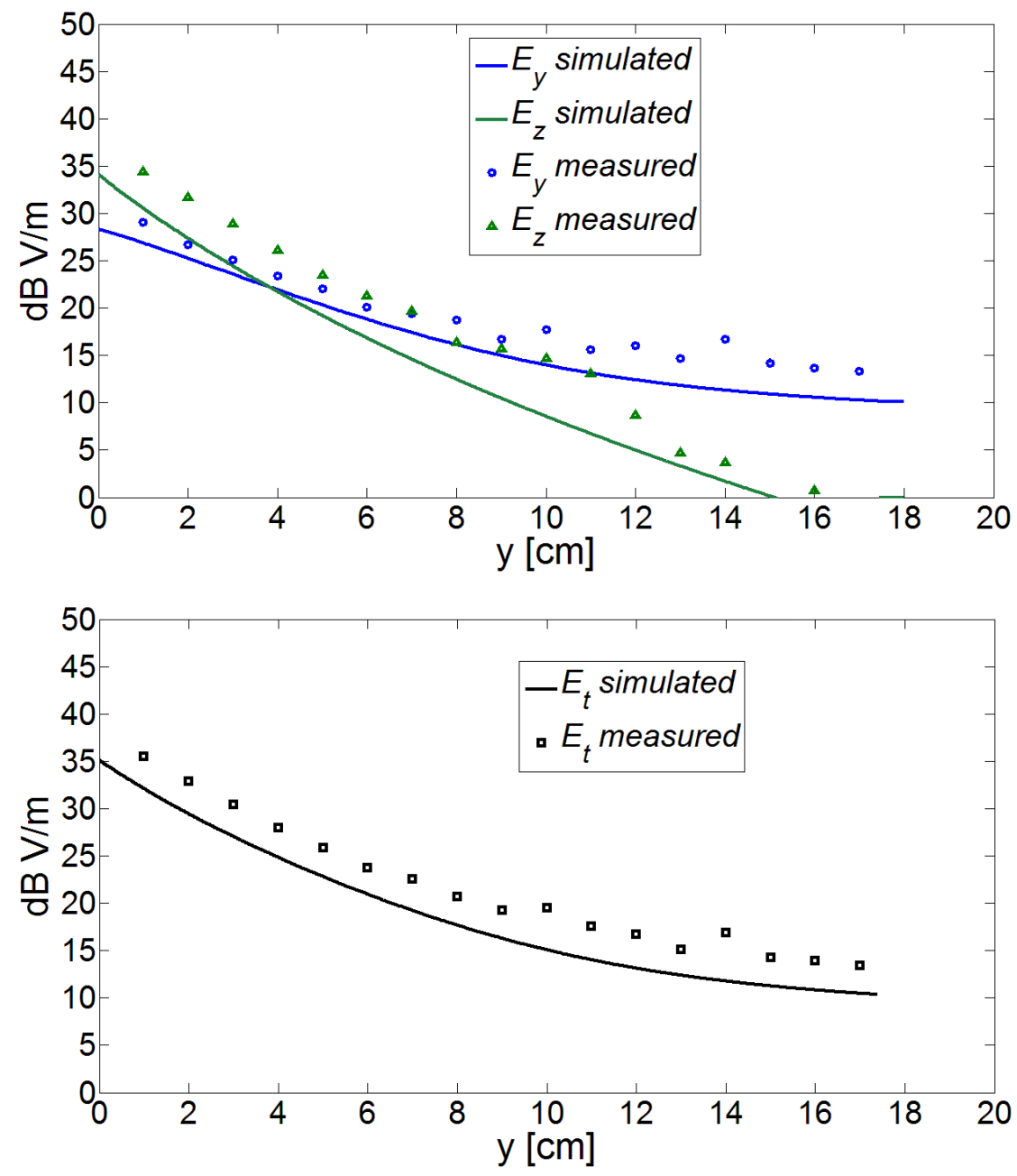

Fig. 10 Measured and simulated near-field components at plane $x=0, x=3$. 


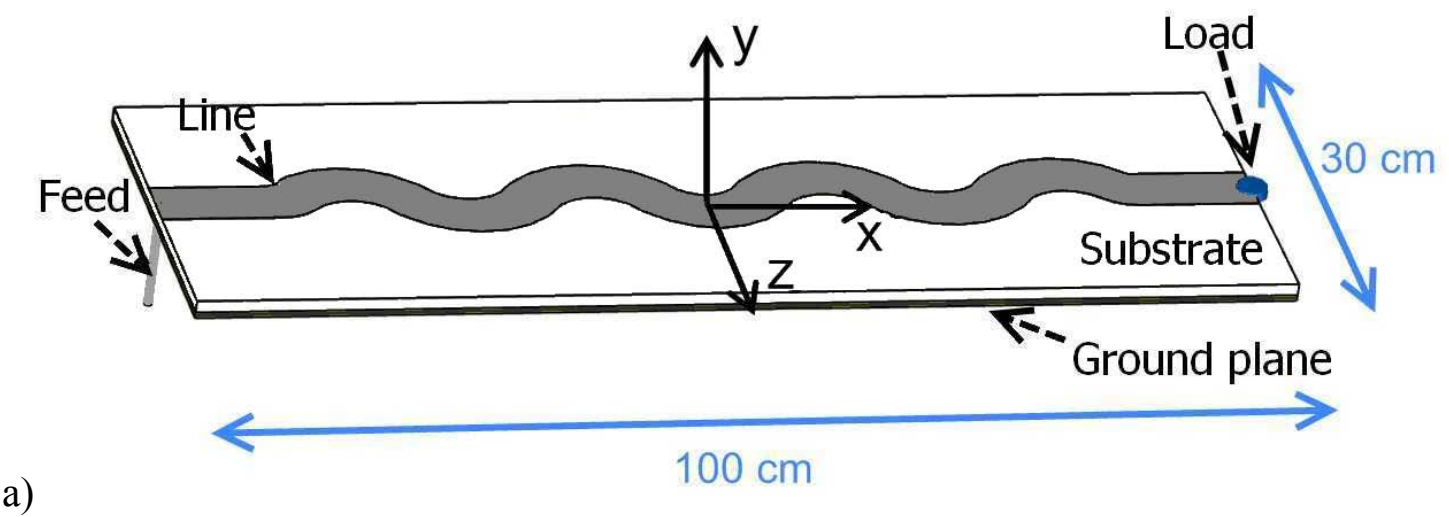

a)

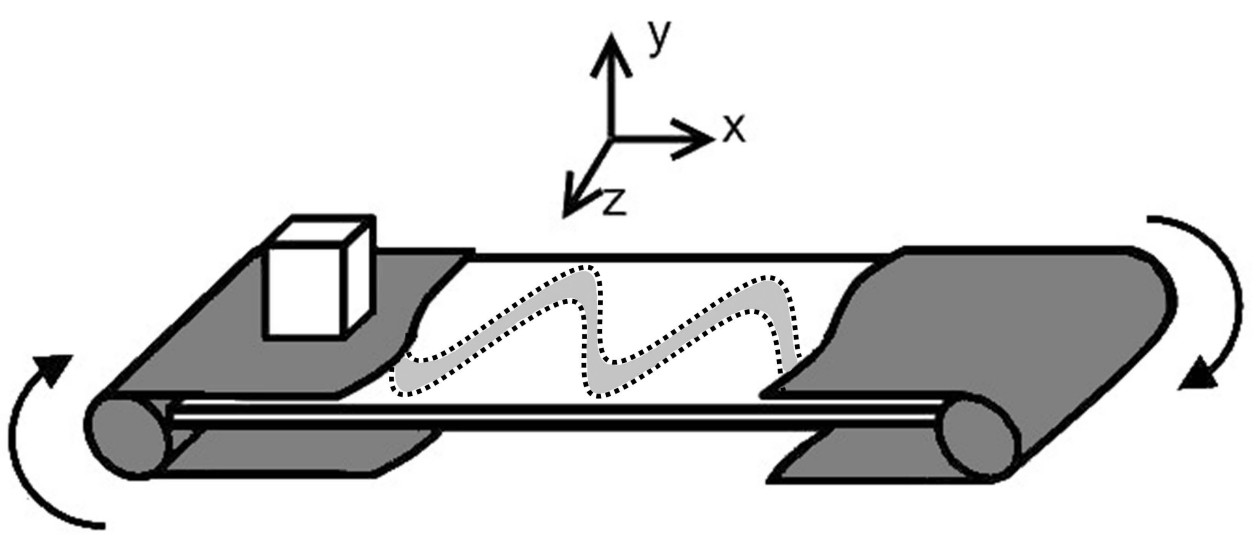

Fig. 11 RFID smart conveyor belt configuration: (a) meandered microstrip line; (b) assembly. 

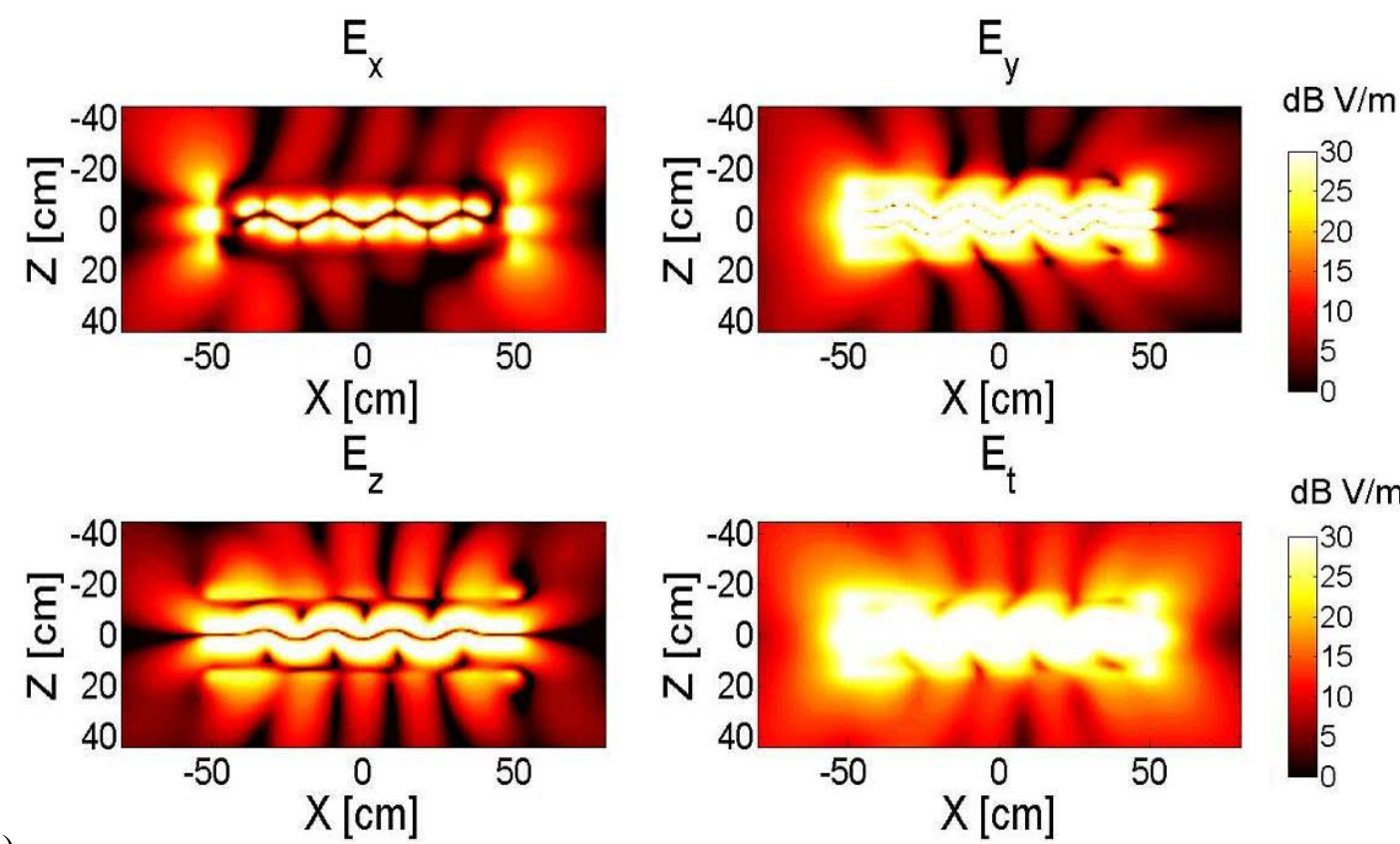

a)
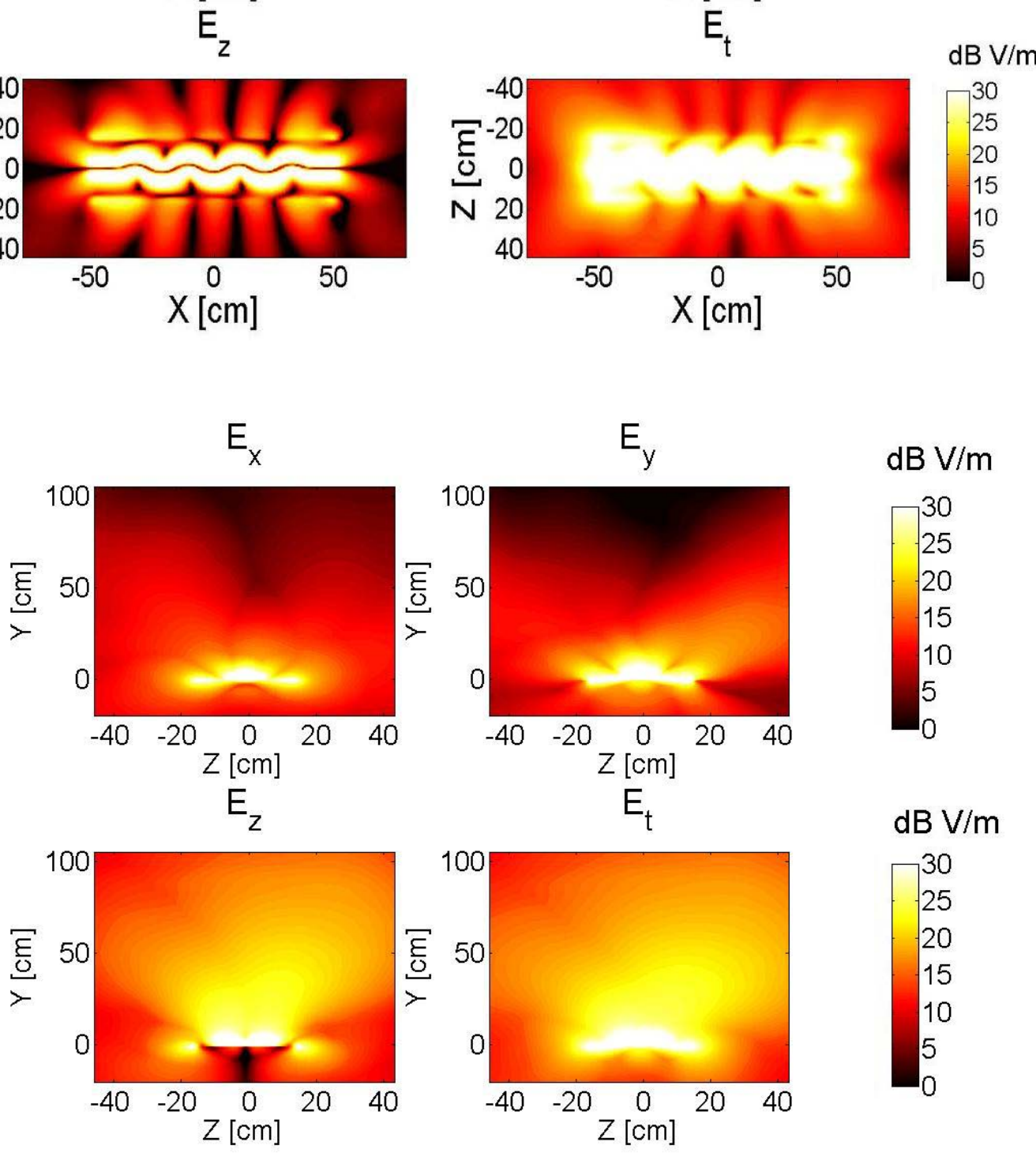

$\mathrm{dB} \mathrm{V/m}$
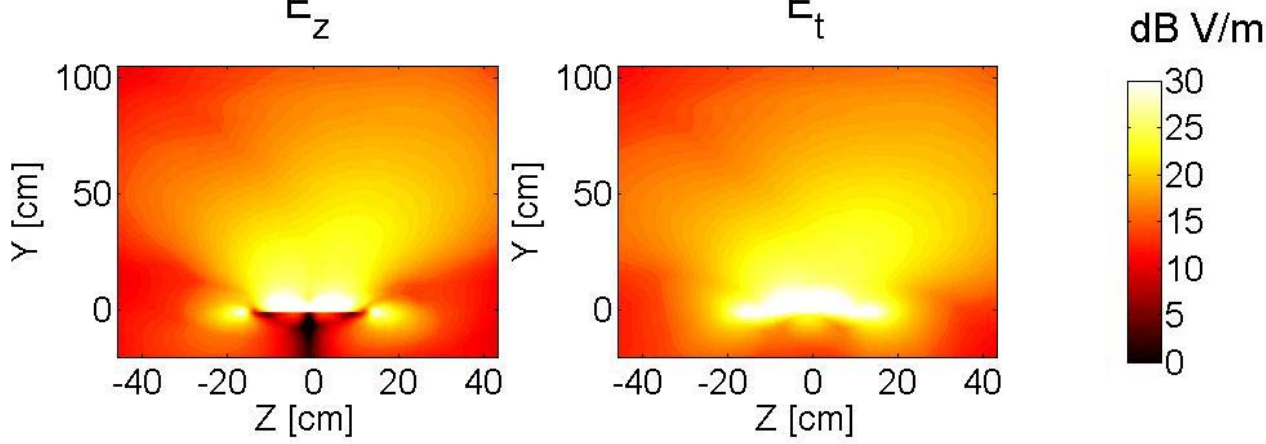

b)

Fig. 12 Simulated empty conveyor belt near-field components $\left(E_{x}, E_{y}\right.$ and $\left.E_{z}\right)$ and total near-field $\left(E_{t}\right)$ amplitude at $866 \mathrm{MHz}$ : (a) $x z$ plane, (b) cumulative maximum of the E-field in the $y z$ plane for $-50 \mathrm{~cm}<\mathrm{x}<50$ cm scan. 


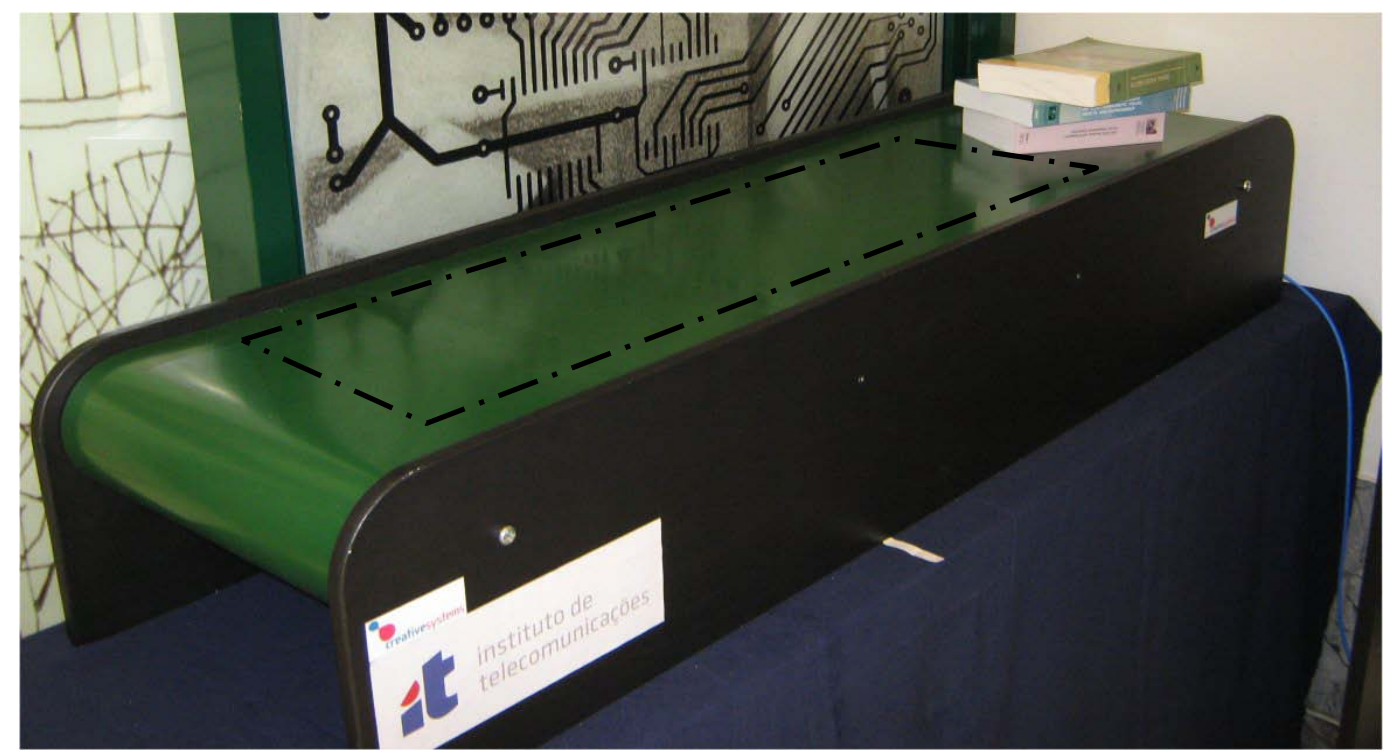

Fig. 13 Fabricated conveyor belt prototype with embedded meandered microstrip line (located beneath the belt in the region bounded by the dashed line).

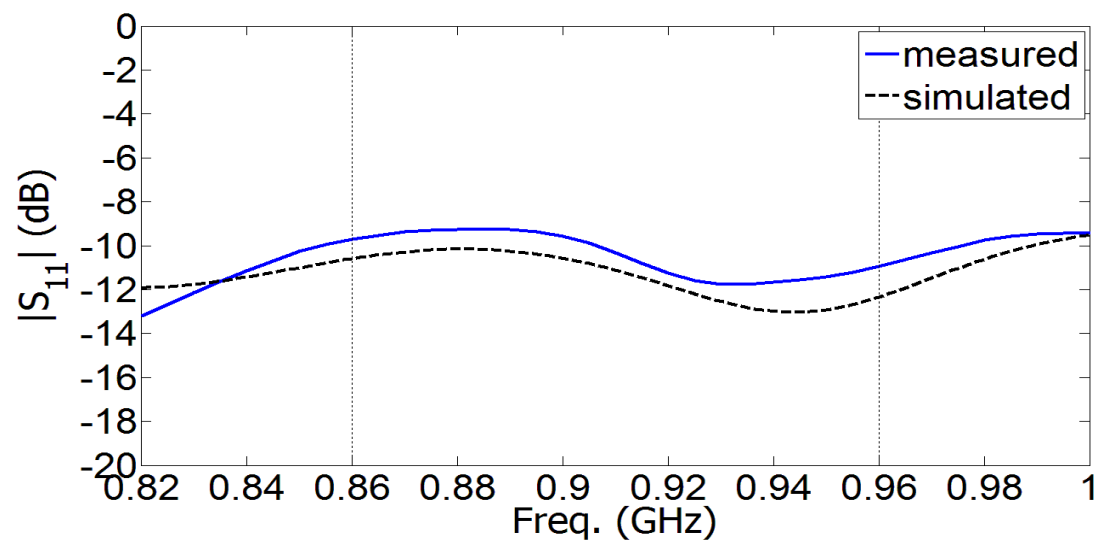

Fig. 14 Measured and simulated input reflection coefficient amplitude of the conveyor belt radiating structure.

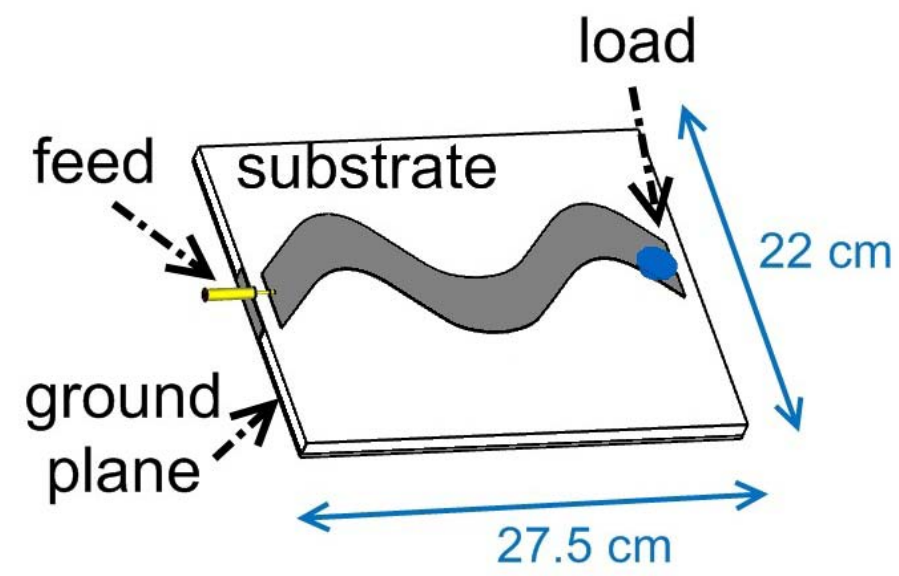

Fig. 15 RFID isolated point reader configuration. 

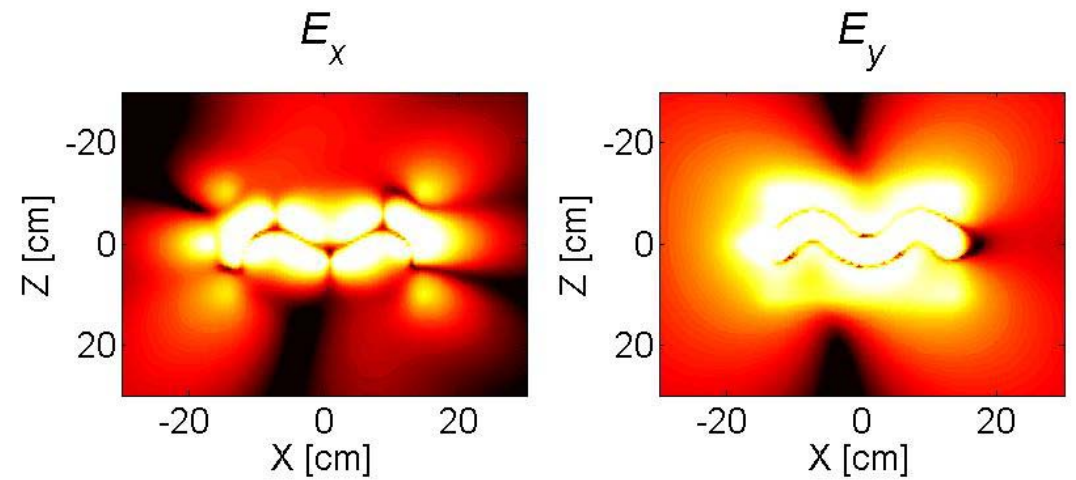

$\mathrm{dB} V / \mathrm{m}$
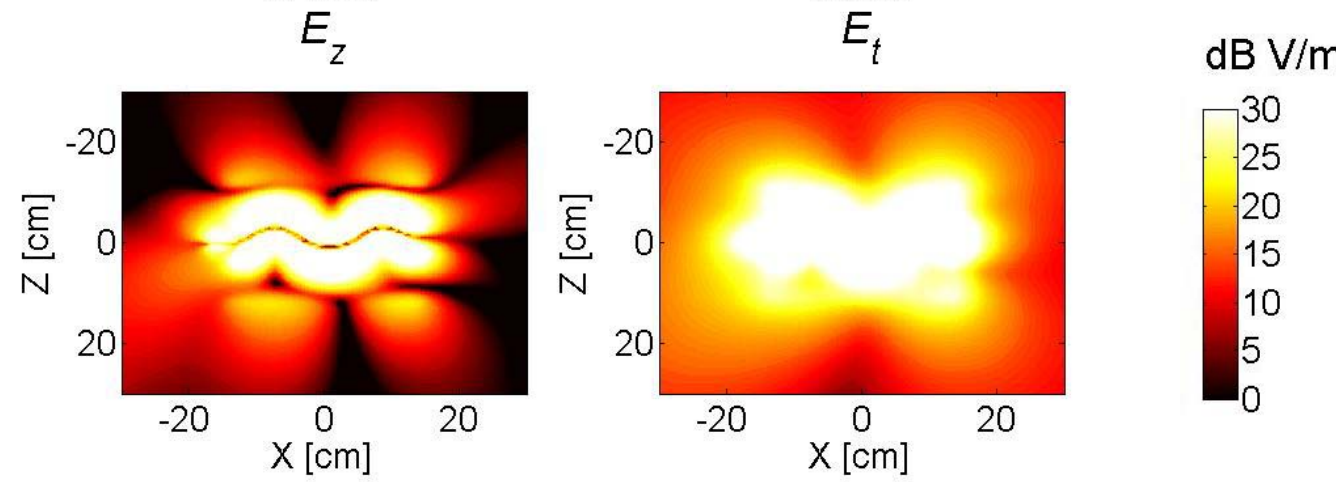

Fig. 16 Simulated empty point reader $x z$ plane near-field components $\left(E_{x}, E_{y}\right.$ and $\left.E_{z}\right)$ and total near-field $\left(E_{t}\right)$ amplitude at $866 \mathrm{MHz}$.

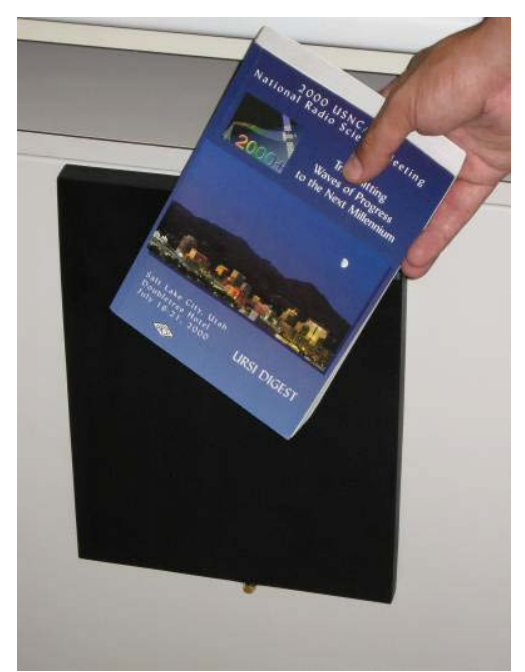

Fig. 17 Fabricated point reader prototype (black box) with embedded meandered microstrip line. 

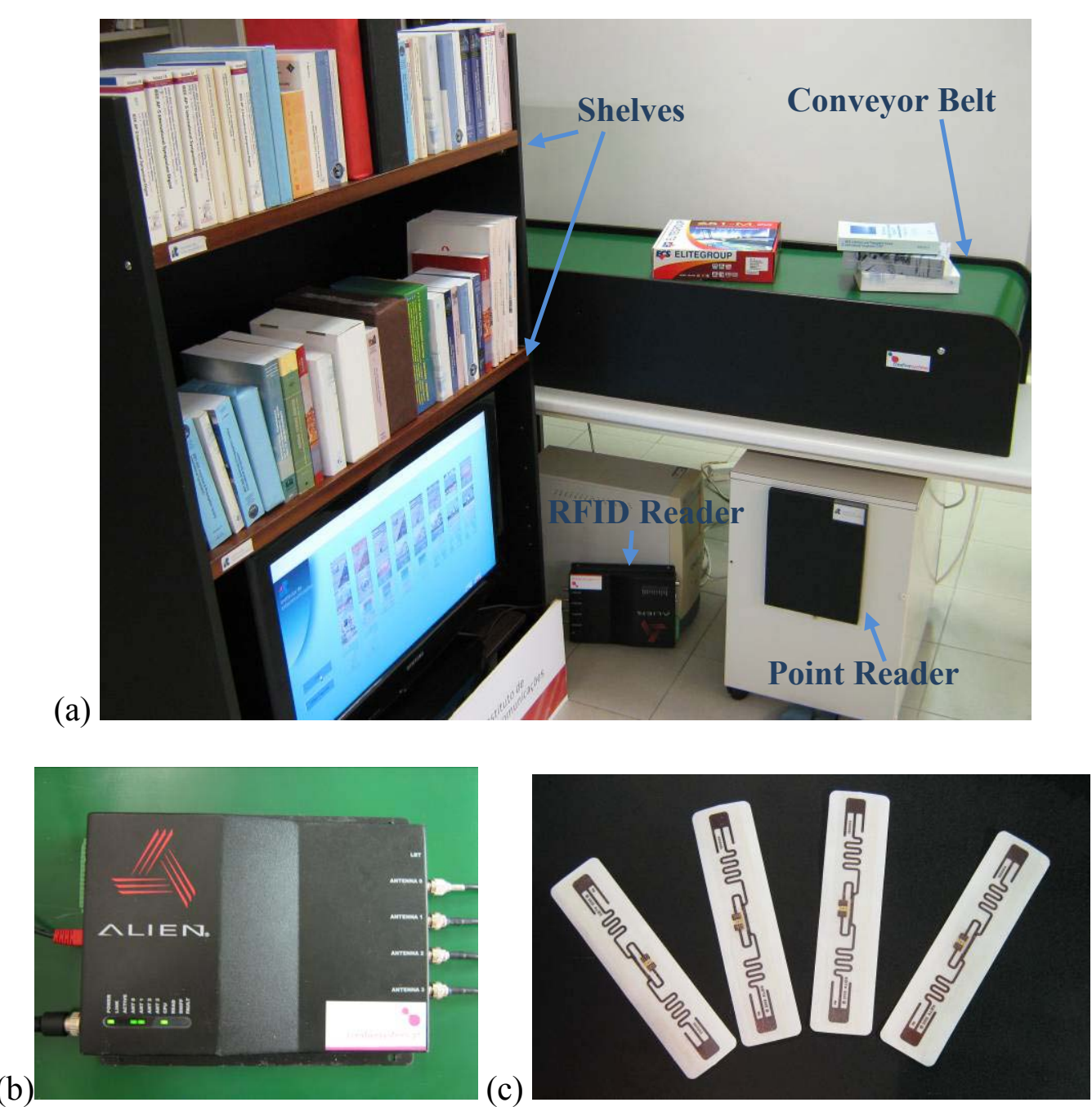

Fig. 18 (a) Test Environment; (b) ALIEN ALR-8800 reader; (c) ALIEN ALN-9540 - "Squiggle" tag. 


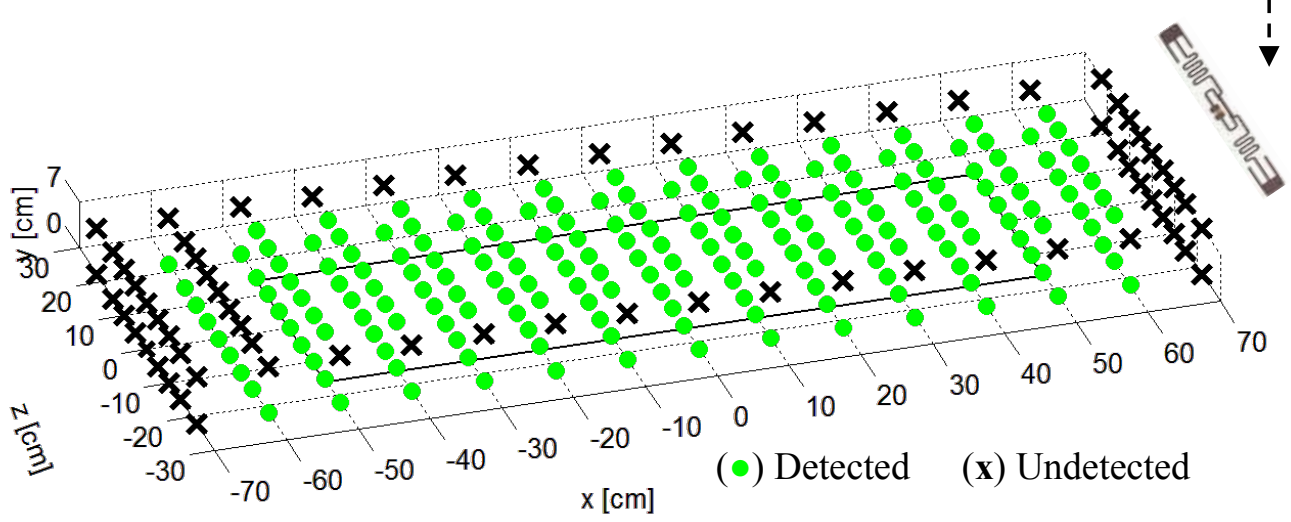

Fig. 19 Measured detection score of a standalone tag oriented along z-axis that scans the volume above the shelf without books.

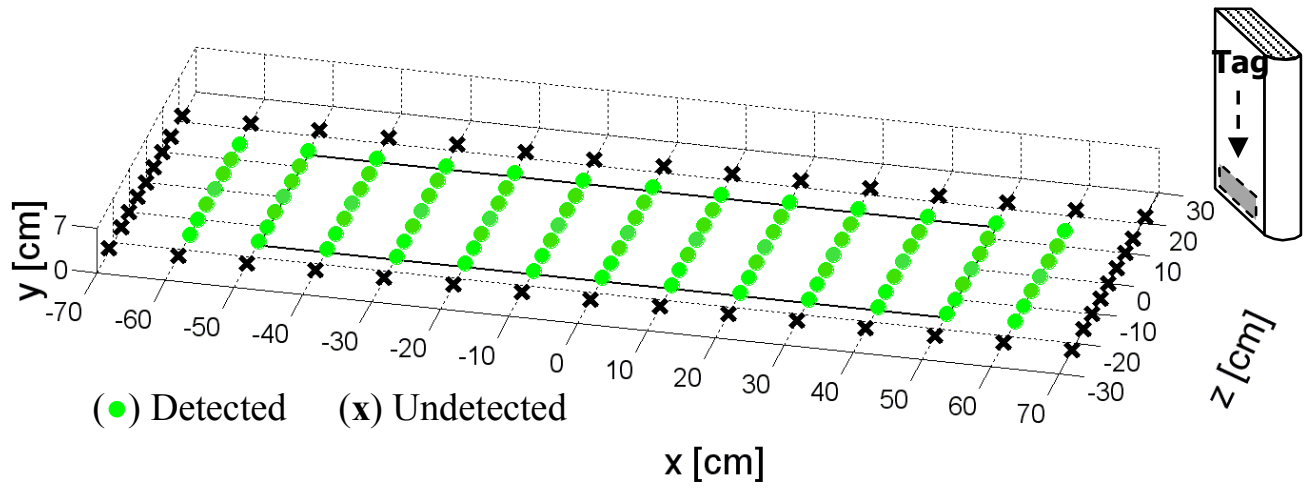

a)
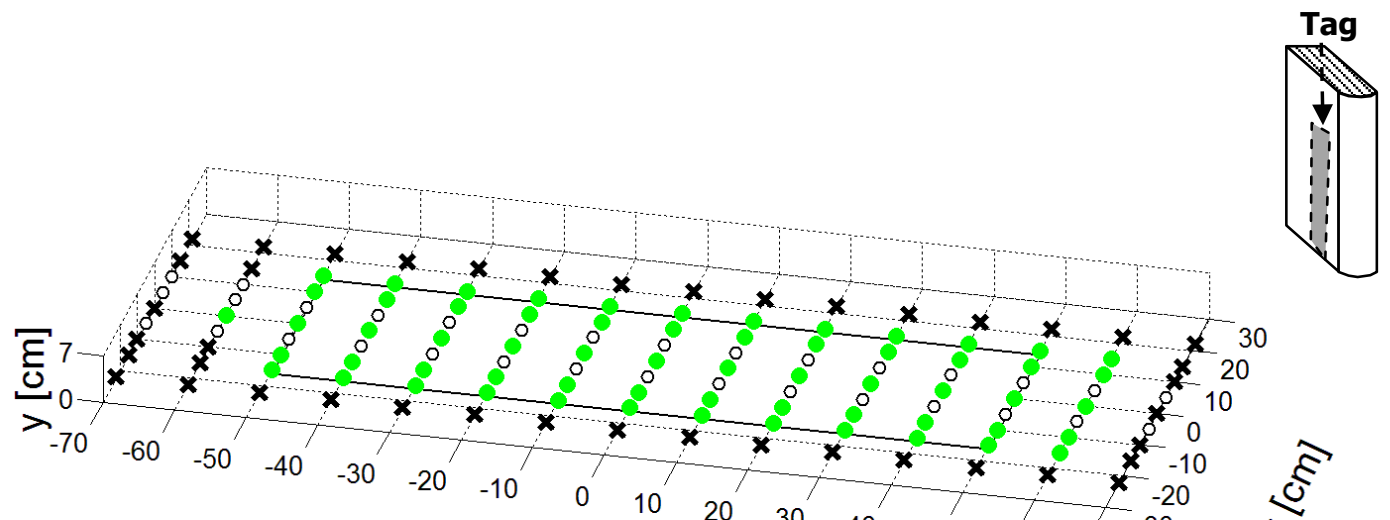

$(\bullet)$ Detected (x) Undetected

(o) Intermittent

$\mathrm{x}[\mathrm{cm}]$

b)

Fig. 20 Measured detection score of a tag placed inside a book at different positions in the volume above the shelf: (a) tag is oriented along z-axis; (b) tag is oriented along y-axis. 

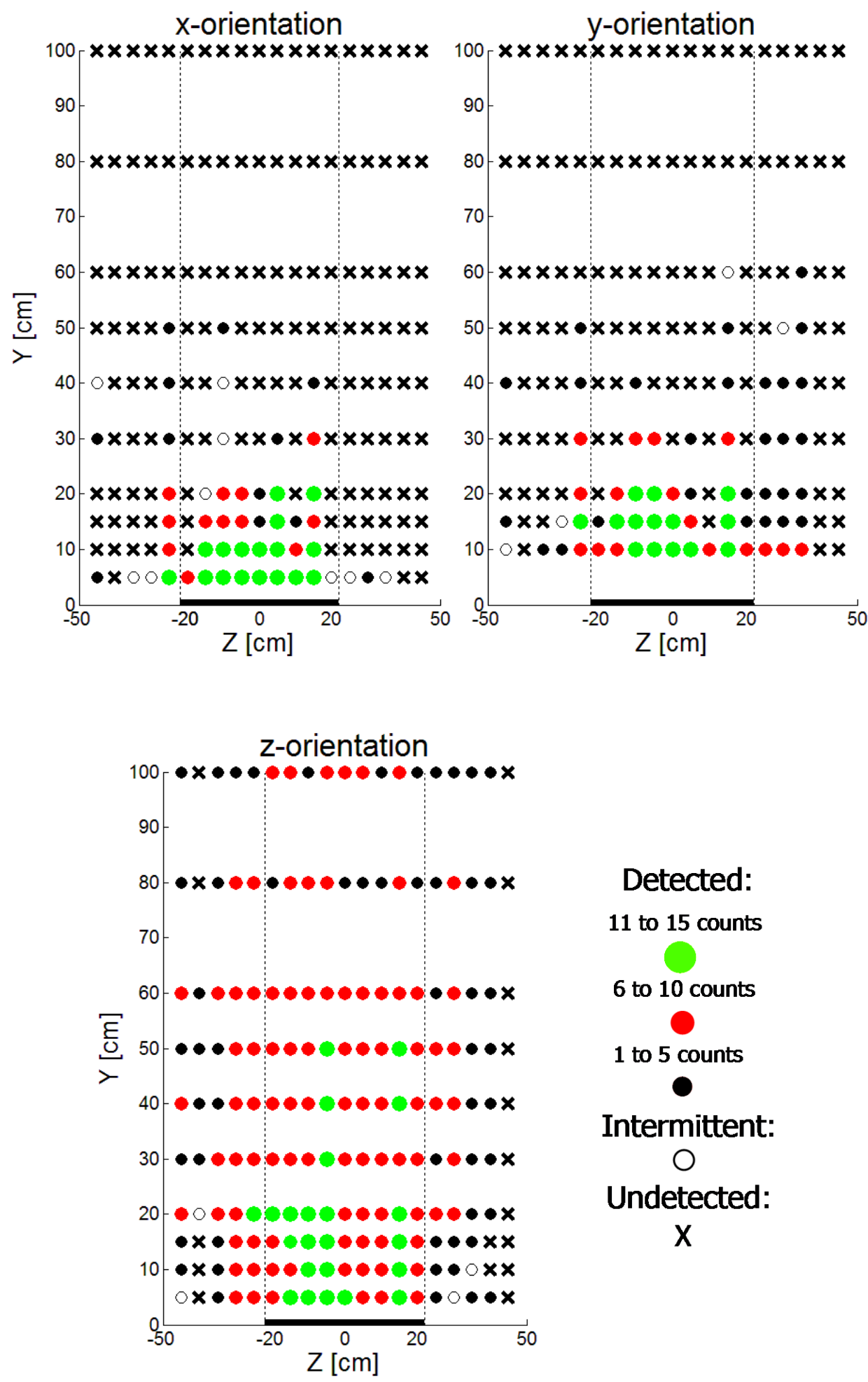

Detected:

11 to 15 counts

6 to 10 counts

.

1 to 5 counts

○

Intermittent:

$O$

Undetected:

$\mathrm{X}$

Fig. 21 Experimental reading score of tags positioned at each represented point after $\mathbf{x}$-axis sliding over the belt. Filled circles represent full detection. Fill color represents detection redundancy. 